TI 2013-027/VII

Tinbergen Institute Discussion Paper
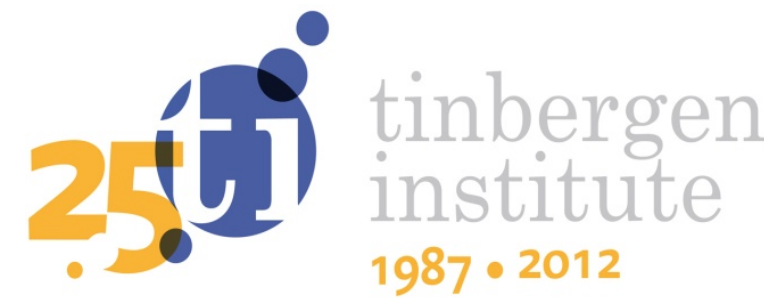

\title{
Gaming in Combinatorial Clock Auctions
}

\author{
Maarten Janssen 1,3 \\ Vladimir Karamychev2,3
}

1 University of Vienna;

2Erasmus School of Economics, Erasmus University Rotterdam;

3 Tinbergen Institute. 
Tinbergen Institute is the graduate school and research institute in economics of Erasmus University Rotterdam, the University of Amsterdam and VU University Amsterdam.

More TI discussion papers can be downloaded at http://www.tinbergen.nl

Tinbergen Institute has two locations:

Tinbergen Institute Amsterdam

Gustav Mahlerplein 117

1082 MS Amsterdam

The Netherlands

Tel.: +31(0)205251600

Tinbergen Institute Rotterdam

Burg. Oudlaan 50

3062 PA Rotterdam

The Netherlands

Tel.: +31(0)10 4088900

Fax: $+31(0) 104089031$

Duisenberg school of finance is a collaboration of the Dutch financial sector and universities, with the ambition to support innovative research and offer top quality academic education in core areas of finance.

DSF research papers can be downloaded at: http://www.dsf.nl/

Duisenberg school of finance

Gustav Mahlerplein 117

1082 MS Amsterdam

The Netherlands

Tel.: +31(0)20 5258579 


\title{
Gaming in Combinatorial Clock Auctions ${ }^{\dagger}$
}

\author{
Maarten Janssen and Vladimir Karamychev \\ University of Vienna \\ Erasmus University Rotterdam and Tinbergen Institute
}

This version: $16-12-2013$

\begin{abstract}
Combinatorial Clock Auctions (CCAs) have recently been used around the world to allocate spectrum for mobile telecom licenses. CCAs are claimed to significantly reduce the scope for gaming or strategic bidding. This paper shows, however, that CCAs facilitate strategic bidding. Real bidders in telecom markets are not only interested in the spectrum they win themselves and the price they pay for that, but also in raising rivals' cost. CCAs provide bidders with excellent opportunities to do so. High auction prices in recent auctions in the Netherlands and Austria are probably to a large extent due to the CCA format. Bidding under a budget constraint is also a highly complicated gaming exercise in a CCA.
\end{abstract}

Key Words: Combinatorial auctions, Telecom markets, Raising rivals’ cost.

JEL Classification: D440, L960.

\footnotetext{
† We have benefited from comments by Martin Bichler, Eric van Damme, Jacob Goeree, Paul Klemperer, Jon Levin, Emiel Maasland, Tuomas Sandholm, David Salant, Andrzej Skrzypacz, and Achim Wambach on earlier versions of this paper, and seminar participants in Vienna, Moscow, Rotterdam, London, Cologne, and Zurich.
} 


\section{Introduction}

Combinatorial clock auctions (CCAs) are used around the globe to allocate mobile telecom licenses among interested bidders. The CCA has a first clock phase where bidders can express demand at increasing prices, and a second supplementary round, which is sealed-bid. The CCA approach has recently been (or will soon be) used in Australia, Canada, and in many European countries (such as Switzerland, Ireland, The Netherlands, Austria, and the United Kingdom). Thus, CCA seems to have superseded the more traditional simultaneous ascending auction (SAA) that until recently was the predominant auction form in telecom auctions. The advantage of the CCA is that it takes package bidding seriously. The CCA is presented to national authorities as a relatively complicated auction model that, if well understood, simplifies bidding as the scope for strategic bidding or "gaming”, in the sense of taking risks to manipulate outcomes, is rather limited (see, e.g., Cramton, 2012). ${ }^{1}$ The possibility of strategic bidding is considered to be one of the main deficiencies of a SAA (see, e.g., Grim et al., 2003).

This article shows, however, that the CCA induces bidders to bid strategically and put them in a position where risky choices have to be made, where the risk is endogenous to the auction. This may harm not only bidders, who may win undesirable outcomes, but also social welfare if the spectrum is allocated in such a way that unusable packages are allocated.

CCA is developed for markets where bidders only attach a value to their own package and the price they pay (see, e.g., Ausubel, Cramton, and Milgrom, 2006). In that case, bidding truthfully constitutes one of the possible equilibrium behaviors (Levin and Skrzypacz, 2013). However, in many auctions (such as those for telecom spectrum) companies are likely

${ }^{1}$ For example, in the abstract of his paper, Cramton (2012) says, "the pricing rule and information policy [of a CCA auction, MJ and VK] are carefully tailored to mitigate gaming behavior". The Irish regulator Regcom (2012, p. 70) states that their consultancy firm DotEcon notes, "the second price rule is utilized to disincentivize gaming behavior and encourage straightforward bidding”. 
to be also interested in how much competitors pay for their spectrum. ${ }^{2,3}$ After acquiring spectrum, winners have to invest large sums of money in developing or upgrading a network. Given imperfect capital markets, it is likely that the more bidders pay for their licenses, the more expensive it becomes to finance their future investments. This means that firms are interested in raising rivals' costs by increasing the price others pay for their licenses. Another reason why firms' behavior is affected by raising rivals' cost motives relates to governance issues within a firm. The information released during and at the end of the auction is such that the only way senior management (or shareholders and/or market analysts) can evaluate the success of a firm's bid strategy is to compare the packages different firms obtain and the prices they pay. A bid strategy is easily considered as unsuccessful if another firm paid much less for objectively better spectrum. Therefore, it is important to raise rivals' cost. ${ }^{4}$

We introduce the preference for raising rivals' cost in a lexicographic way, i.e., this preference only affects bidders' decisions in case the surplus for their winning package remains the same. ${ }^{5}$ Thus, our treatment of raising rivals' cost can be regarded as a robustness check on the standard results regarding CCAs. One implication of modeling raising rivals' cost in this way is that bidders will never let the raising rivals' cost motive prevail over the primary objective of winning a package at a price below value. In VCG auctions where given the behavior of others, a bidder is indifferent between many possible strategies under the

2 That firms are likely interested in other winners paying more for their licenses is confirmed in recent policy discussions (for instance OFCOM, 2012, page 122, point 7.9, for the United Kingdom).

${ }^{3}$ As the value of spectrum also depends on how many winners the auction has, their identity (whether they are incumbents or entrants), and on the quality of the package of licenses the competitors get, bidders valuation may actually be endogenous to the auction outcome. The literature on this issue (see, e.g., Goeree, 2003; Jehiel and Moldovanu, 2000, 2003; Jehiel, Moldovanu, and Stacchetti, 1996; Janssen and Karamychev, 2007, 2010; Klemperer, 2002a, 2002b) does not consider CCAs, and we do not consider this issue in much detail (apart from a short discussion in the concluding section).

4 The Swiss auction outcome shows that payments for similar spectrum can be quite unequal. See BAKOM (2010) for the Swiss auction design, and BAKOM (2012) for the outcome.

5 There is a reasonably large literature on auctions with financial externalities, or auctions with a "spite motive" (see, e.g., Cooper and Fang, 2008; Morgan et al., 2003; Maasland and Onderstal, 2007; Shandra and Sandholm, 2010; and recently Fiat et al., 2012; and Lu, 2012). This literature typically deals with the standard single object auction, and asks the question how bidding behavior is affected if bidders also care about what the winner pays (even if they are not winners themselves). Our paper differs from this literature in that in a multi-object auction, bidders can be winners and still raise rivals' cost of other winners by placing bids on packages they themselves are not winning. This complicated gaming aspect is not present in single object auctions. 
standard objective function (as they all result in the same allocation and the same price they pay), lexicographic preferences solve the indifference in favor of strategies raising rivals' cost (and improving relative performance).

We show that if firms have a lexicographic preference for raising rivals' cost, the Vickrey-Clark-Groves (VCG) mechanism underlying the CCA does not have a (weakly) dominant strategy. Nevertheless, under additional conditions, the CCA, unlike the VCG mechanism, can be solved using iterative elimination of (weakly) dominated strategies. In the resulting equilibrium, bidders express aggressive bids above value on some packages, and they may even bid on packages without intrinsic value. Bidding truthfully is an iteratively dominated strategy. How much to bid above value is the first gaming aspect in CCAs.

In October 2013 after raising a very high revenue of 2 billion euro's for a country of 8 million inhabitants, the Austrian regulator RTR felt compelled to reveal an overview of the bidding behavior of the three participating bidders. The behavior is fully in line what this paper predicts (see, https://www.rtr.at/en/pr/PI28102013TK):

"The three bidders actually submitted a total of more than 4,000 supplementary bids. More than 65\% of these supplementary bids were submitted for the largest permissible combinations of frequency blocks, with a share of some 50\% of available frequencies. In addition, the bidders utilised almost to the full the price limits that had applied to these large packages during the sealed-bid stage. ... These supplementary bids submitted on large frequency packages had a significant effect on the prices offered by the other bidders. At the same time, such bids generally only have a marginal likelihood of winning out in the end. If these bids for very large numbers of frequencies had been ignored when determining the winners and prices, the revenue from the auction would have settled at a level of about EUR 1 billion”.

Another important aspect of real-world telecom auctions is that bidders have limited budgets. In regular second-price auctions for one unit, budget constraints do not impose serious challenges as bidders can simply bid up to the minimum of their budget and their valuation. In case of multiple objects, such a strategy can lead, however, to extreme inequality in 
payments, as a budget constraint bidder cannot express higher bids for larger packages (which is an important component in the determination of what other bidders have to pay).

When bidders have a preference for raising rivals’ cost, budget constraints impose severe challenges for determining optimal bid strategies in a multi-unit context. First, it is not obvious what the notion of bidding under a budget constraint means. There are at least three interpretations that are possible: (i) do not place any bid above budget, (ii) only place bids above budget on packages that - given the development of the auction so far - cannot be winning, and (iii) place bids (above budget) such that equilibrium payments are not above budget. Second, bidders that stick to the first and second interpretation of a budget constraint run the risk of having to pay more for identical packages than competitors adhering to the third interpretation of a budget constraint. Bidding under a budget constraint can then lead to multiple equilibria with a Hawk-Dove type flavor: more aggressive bidders perform well if they play against less aggressive bidders, but their bidding can also lead to payments above budget. Bidding under a budget constraint is the second gaming aspect discussed in this paper.

A recent working paper by Levin and Skrzypacz (2013) is close in spirit to our paper. ${ }^{6}$ Using the fact that with intrinsic preferences only, a VCG mechanism has multiple equilibria, they show that the interaction between early phases of the CCA and the final sealed-bid round, which essentially is a VCG auction, depends on how people resolve indifferences. Final allocations and prices in a CCA may then be quite different from the VCG prices. In a two-bidder model with one divisible commodity, they show that a lexicographic preference for raising rivals' cost may result in demand reduction strategies in early phases of the CCA. Our main focus is on bidder behavior in the final sealed-bid round with multiple units of different commodities. A more detailed discussion of this and some other differences is contained in Sections 3 and 4.

6 A first draft of Levin and Skrzypacz (2013) has become available in September 2013. A first working paper version of this paper (Janssen and Karamychev, 2013) has been publicly available in February 2013. 
There are some other recent papers also shedding some critical light on CCAs. Goeree and Lien (2012) show that the core selection principle introduced in the pricing rule used in CCAs implies that bidding valuation is no longer an optimal bidding strategy. Beck and Ott (2011) show that this principle may imply that bidding both above and below valuations can be optimal in CCAs. Knapek and Wambach (2012) show that bidding in a CCA may be strategically complicated. Bichler et al. (2013) present some experimental results on CCA and show that CCA's may lead to inefficient outcomes. They attribute the inefficiency relative to SAA to the so-called missing bids problem. In the Appendix, they also provide an example of spiteful bidding in a CCA. ${ }^{7}$ None of these papers provide, however, a general analysis of how bidders can game in the auction to strategically raise rivals' cost. Moreover, they also do not analyze the implications of budget constraints on bidding behavior.

The rest of the paper is organized as follows. Section 2 provides a more detailed description of the different stages of the CCA. Section 3 discusses how rational bidders will bid in a CCA if the only deviation from the traditional auction set-up is that bidders have a preference for raising rivals' cost. Section 4 presents an extensive example illustrating how a CCA works and showing how large the deviation from truthful bidding can be if bidders have only a tiny preference to raise rivals' cost. Section 5 introduces budget constraints, in addition to bidders’ preference for raising rivals’ cost. Section 6 concludes with a discussion. The appendix contains all proofs and additional information on CCAs.

\section{Combinatorial Clock Auctions}

Most recent spectrum auctions allocate spectrum in different frequency bands. In each frequency band, the spectrum is divided in a certain number of blocks. Let there be $K$ different frequency bands with $n_{k}$ blocks in band $k$. Spectrum in different frequency bands has different properties in terms of geographic (or indoor) coverage and capacity. Mobile

${ }^{7}$ Salant (2013) provides an informed overview of some recently used auction design and a discussion on some outcomes of CCAs that have been held recently. 
telecom companies, therefore, want to acquire a mix of spectrum in different frequency bands. The set of all possible (and feasible) packages is denoted by $\Pi$, and the aggregate supply is denoted by $\bar{\pi}, \bar{\pi} \equiv\left(n_{1}, \ldots, n_{K}\right)$.

A combinatorial clock auction allocates the available spectrum using two integrated phases. $^{8}$ The first phase is a clock phase, which is divided into several clock rounds. In each round $t$, the auctioneer announces clock prices $p^{t}=\left(p_{1}^{t}, \ldots, p_{K}^{t}\right)$, one price for each frequency band. The (reserve) prices in the first round $p^{1}$ are set in advance of the auction. At these given prices, bidders express how many blocks they would like to acquire in each band. The number of blocks in band $k$ demanded by player $i$ in round $t$ is denoted by $d_{i, k}^{t}$, and the whole package that player $i$ demands in round $t$ is denoted by $d_{i}^{t}=\left(d_{i, 1}^{t}, \ldots, d_{i, K}^{t}\right) \in \Pi$.

At the end of a round, bidders are typically only informed about the total demand in each band. $^{9}$ If, in round $t$, there is excess demand in band $k$, clock prices for that band will increase in clock round $(t+1)$ by a predetermined price increment $\delta_{k}>0: p_{k}^{t+1}=p_{k}^{t}+\delta_{k}$. The clock phase of the auction stops when there is no excess demand in any band. The intention of the clock phase is to assist bidders in discovering what the prices of the different frequency bands could be (Ausubel, Cramton and Milgrom, 2006). The clock phase also serves to impose restrictions on what bidders can bid in the second phase, the supplementary round. At the end of the clock phase, no allocation of spectrum takes place yet. ${ }^{10}$

Each block in a spectrum band is given a certain number of so-called eligibility points. These points are used to provide a one-dimensional measurement of a player's demand. If a block in band $k$ requires $e_{k}$ eligibility points, $e=\left(e_{1}, \ldots, e_{K}\right)$, then the total eligibility points of player $i$ 's demand in a round is given by $E_{i}^{t}=e \cdot d_{i}^{t}=\sum_{k=1}^{K}\left(e_{k} d_{i, k}^{t}\right)$. In any clock round, a bidder cannot express a demand that requires a larger number of eligibility points than the

${ }^{8}$ Spectrum auctions typically have a third (assignment) phase where specific location in a spectrum band is allocated. As this is of no concern for our paper, we will not discuss this assignment phase.

${ }^{9}$ In the CCA that was held in Austria in 2010, even that information was not available to bidders. Bidders only knew that there was excess demand in a certain band, as the clock prices in these bands (and only in these bands) increased from this clock round to the next round.

10 The Romanian auction in 2012 had some features of a CCA. However, in that auction, frequencies were already allocated to bidders at the end of the clock phase (at the then prevailing clock prices). 
bid expressed in the previous round, i.e., $E_{i}^{t} \leq E_{i}^{t-1}{ }^{11}$ If bidder $i$ expresses a demand for a package in round $t$ that requires a strictly smaller number of eligibility points than the bid in the previous round, i.e., if $E_{i}^{t}<E_{i}^{t-1}$, then that round is called an anchor round. Anchor rounds, the band prices in these anchor rounds, and anchor bids play an important role in determining which bids a bidder can express in the supplementary round.

The supplementary round is a simultaneous bid round, where in one round bidders can express a bid for all possible packages, subject to some constraints. The most commonly used constraint is the so-called relative cap, ${ }^{12}$ which works as follows. ${ }^{13}$

We use the Greek letter $\pi$ to denote generic packages, $\pi=\left(\pi_{1}, \ldots, \pi_{K}\right) \in \Pi$. On the package $\pi^{f}=d_{i}^{T}$ that bidder $i$ was bidding for in the final clock round $T$ (and only on this package), this bidder is unconstrained in the supplementary round. Let us denote the bid expressed for this package in the supplementary round by $b^{f}$. For all other packages, a bidder is constrained, and the constraints are calculated relative to $b^{f}$ as follows. For all packages that require a number of eligibility points $E$ that is smaller than or equal to the number of points $E_{i}^{T}$, the final clock round $T$ is the anchor round. Denoting the final clock round prices by $p^{T}=\left(p_{1}^{T}, \ldots, p_{K}^{T}\right)$, the maximum bid $B^{\alpha}$ in the supplementary round that can be expressed on package $\pi^{\alpha}=\left(\pi_{1}^{\alpha}, \ldots, \pi_{K}^{\alpha}\right)$ is

$$
B^{\alpha}=b^{f}+p^{T} \cdot\left(\pi^{\alpha}-\pi^{f}\right)=b^{f}+\sum_{k=1}^{K}\left[p_{k}^{T}\left(\pi_{k}^{\alpha}-\pi_{k}^{f}\right)\right] .
$$

In order to compute the cap for a package $\pi^{\beta}$, which requires the number of eligibility points $e \cdot \pi^{\beta}$, and for which $e \cdot \pi^{\beta}>E_{i}^{T}$, one first has to track the last round $r$ in the clock phase when bidder $i$ had enough eligibility points to bid for package $\pi^{\beta}$. This $r$ is uniquely determined by:

$$
E^{r}<e \cdot \pi^{\beta} \leq E^{r-1}
$$

11 In certain proposals, this constraint is relaxed into a Simplified Revealed preference with an Eligibility-Point Safe Harbor, see Ausubel and Cramton (2011).

12 An alternative, the final cap rule is described in the Appendix, part II.

13 This constraint is used in auctions in Austria (2010), Switzerland (2012), Ireland (2012), The Netherlands (2012), and United Kingdom (2013), among others. 
Round $r$ is the anchor round for package $\pi^{\beta}$. The maximum price bidder $i$ can then bid for package $\pi^{\beta}$ can be computed iteratively as follows, starting from the final clock round $r=T$. Let $\pi(r)$ be the package bidder $i$ has bid for in clock round $r, \pi(r)=d_{i}^{r}$, and let $b(r)$ be the highest actual bid in the clock or supplementary round expressed for package $\pi(r)$. Then, the maximum bid $B^{\beta}$ that can be expressed on package $\pi^{\beta}$ is

$$
B^{\beta}=b(r)+p^{r} \cdot\left(\pi^{\beta}-\pi(r)\right)=b(r)+\sum_{k=1}^{K}\left[p_{k}^{r}\left(\pi_{k}^{\beta}-\pi_{k}(r)\right)\right] .
$$

Thus, the relative cap expresses upper bounds on what bidders can bid for certain packages relative to their bid for the package they were bidding for in the final clock round. The relative cap has a clear economic interpretation in terms of revealed preference (see, e.g., Ausubel, Cramton, and Milgrom, 2006).

Finally, we explain how the winners of the auction and the final auction prices are determined. The winners are determined in the same way as in the VCG mechanism. Of all feasible combinations of bids, one per bidder, a combination is selected that maximizes the total sum of the bids. Bids that are parts of this combination, are winning bids, and bidders who have submitted these bids win their winning bids' packages. The CCA prices are equal to the VCG prices, if the VCG prices are in the core. If the VCG prices are not in the core, CCA typically uses some adjustment such that the CCA prices are in the core (see, e.g., Day and Raghavan, 2007, Day and Milgrom, 2008, and Erdil and Klemperer, 2010). As we do not want our arguments to depend on the specific core selection principle that is used (as Goeree and Lien, 2012, have already shown that core-selecting pricing rules imply that it is not optimal to bid straightforwardly), we assume that CCA bids are such that the CCA prices are in the core. In this case, CCA prices coincide with VCG prices.

The VCG price a winner has to pay for the package he wins equals the opportunity cost he imposes on others. Thus, a winner pays the maximum price the other bidders are willing to pay additionally for the spectrum he won. All elements of the description of the CCA are illustrated in the example in Section 4. 


\section{Preference for Raising Rivals' Cost}

We augment an otherwise standard auction model with bidders' preferences for raising rivals' cost. Thus, apart from their own private values drawn from an arbitrary joint distribution, bidders are also interested in driving up prices their competitors have to pay. The joint distribution will not play an important role in our analysis, and we do not model it explicitly. The intrinsic valuation of bidder $i$ for a package $\pi^{\alpha}$ is denoted by $v_{i}^{\alpha}$, and when no confusion is possible we drop subscript $i$.

We model the preference for raising rivals' cost as a lexicographic preference. By the end of the supplementary round, any bidder has expressed a set of bids $\Phi=\left\{\left(b^{\alpha}, \pi^{\alpha}\right): \pi^{\alpha} \in\right.$ $\Psi$ \} on a subset of packages $\Psi \subset \Pi$, where $b^{\alpha}$ is the bid that the bidder exerts on package $\pi^{\alpha}$. A bidder either wins one of these packages $\pi^{\alpha}$ and has to pay $p^{\alpha}$ (which does not depend on own bids, but only on other bidders' bids) or he does not win anything. His intrinsic pay-off of bidding $\Phi$ is thus equal $\left(v^{\alpha}-p^{\alpha}\right)$ or 0 . If, for a fixed strategy profile of other bidders, the intrinsic pay-offs of two strategies are identical, then bidders prefer the strategy that raises the sum of rivals’ payments most. A strategy $\Phi$ (weakly) dominates another strategy $\Phi^{\prime}$ if, for any combination of bids of the other bidders:

1. $\Phi$ never results in a lower intrinsic pay-off, or in the same intrinsic pay-off and a lower sum of rivals' payments than $\Phi^{\prime}$; and

2. For some combination of bid of the other bidders, either $\Phi$ results in a larger intrinsic pay-off, or it results in the same intrinsic pay-off and raises the sum of rivals' payments compared to $\Phi^{\prime}$.

To start the analysis, we first analyze bidding in the supplementary round in case bidders have bid straightforwardly in the clock phase. We show that the supplementary stage does not have a (weakly) dominant strategy, and under certain conditions, an equilibrium in the supplementary round of the CCA can be found using two rounds of iterative elimination of weakly dominated strategies, resulting in bids that differ from the intrinsic valuations. As the elimination procedure uses information concerning maximal bid increments from the clock 
phase, and the VCG mechanism does not have bidding caps, the latter mechanism is not dominance solvable anymore. Going back to the clock phase, bidders find it optimal not to bid according to their intrinsic preferences in the clock phase of the CCA, resulting in higher auction prices.

\subsection{The Supplementary Phase}

For simplicity, we first assume that the development of the clock phase is public information. Then, we show that the qualitative analysis continues to hold, if bidders are only informed about total demand in every clock round, like in most real-world auctions.

The first Proposition states that for any development of the clock phase, each bidder can calculate a so-called "knock out" bid $\widehat{b^{f}}$, KO-bid hereinafter, that is such that if the bidder makes this bid on his final clock round package $\pi^{f}$ then he guarantees himself to win. ${ }^{14}$

Proposition 1. Let all individual clock round bids be public information, and let package $\pi^{f}$ be the final clock round package of bidder $i$. Then, there exist an amount $\widehat{b^{f}}$ such that if bidder $i$ bids $b^{f}>\widehat{b^{f}}$ in the supplementary round on package $\pi^{f}$, he surely wins some package. Moreover, if he wins package $\pi^{f}$, he pays not more than $\widehat{b^{f}}$.

As is explained in Section 2, the bidding behavior of the clock phase imposes restrictions on the bids that are allowed in the supplementary round. If a bidder knows the bidding behavior of the other bidders in the clock phase, he can compute the maximal amount they can bid on any package in addition to the unrestricted supplementary round bid on the final clock round package. This is important information as it imposes an upper bound on what other bidders are allowed to bid more on a combination of packages resulting in the bidder not being part of the winning combination. The "knock out" bid $\widehat{b^{f}}$, is essentially the maximum bid difference the other bidders can generate, given the development of the clock phase, to delete the bidder

14 Previous results for the existence of a knock-out bid have been derived under the much simpler final cap rule (see, e.g., Bichler et al., 2013, and independent work by Ausubel and Cramton, 2011). Most CCA auctions that have been implemented use a relative cap rule as explained before. 
from the winning coalition of bidders. Whether the bidder wins his final clock round package $\pi^{f}$ or some other package depends on the bids the bidder makes on other packages, in combination with other bids of the other bidders. It is interesting to note the difference with the VCG mechanism. In the VCG mechanism, no finite bid guarantees winning any package as bidders in the VCG mechanism are unrestricted in their bids.

The calculations determining the KO-bid make use of the assumption that bidders know the demand of individual bidders in each clock round. However, even if only total demands over all bidders in each clock round are known, a KO-bid can be calculated by considering all possible combinations of individual demands that are consistent with the information concerning total demand and own bidding behavior, and calculating the maximal KO-bid over all those possible combinations. Computationally, this is a much harder problem to solve but, in principle, there is no fundamental difference between the two cases. In Section 4, we present an example showing, among other things, how KO-bids can be calculated.

Suppose now that bidder $i$ has bid sincerely in the clock phase, $\pi^{f}$ is his final clock round package, and $v^{f}$ is his valuation. Using Proposition 1, this bidder can compute his KObid $\widehat{b^{f}}$. The next proposition shows that in a first round of elimination of weakly dominated strategies, a bidder can eliminate all strategies $\Phi$ in which $b^{f}<\min \left\{\widehat{b^{f}}, v^{f}\right\}$.

Proposition 2. Let bidder $i$ bid truthfully in the clock phase. In the supplementary round, all package bids with $b^{f}<\min \left\{\widehat{b^{f}}, v^{f}\right\}$ are weakly dominated.

According to Proposition 2, in the first round of elimination of weakly dominated strategies, all package bids with $b^{f}<\min \left\{\widehat{b^{f}}, v^{f}\right\}$ are eliminated. If $v^{f} \leq \widehat{b^{f}}$, the bidder bids his entire value $v^{f}$ on the package $\pi^{f}$ to increase his chance of winning. If $v^{f}>\widehat{b^{f}}$, by bidding $b^{f}=\widehat{b^{f}}$, the bidder guarantees himself that he is winning some package, and which package he wins depends on the relative bids in combination with bids of other bidders, but not on the absolute amount of the bids. Thus, there is no need to bid more than $\widehat{b^{f}}$ on the final clock round package. The main idea behind Proposition 2 is that if the bidder keeps the same bid 
increments on all packages compared to the package he was bidding for in the final clock round, but increases his bid on the final clock round package, then he will increase his chances of winning. It neither changes the package he wins nor affects the price he pays. As long as the KO-bid is below the value, it always results in a positive pay-off.

We again note the difference with the VCG mechanism. In the VCG mechanism, where bids are not capped and $\widehat{b^{f}}$ is unbounded the bidder should bid his entire value $v^{f}$ on the package $\pi^{f}$. In a CCA, to the contrary, the bidder does not need to bid above $\widehat{b^{f}}$ in case $v^{f}>\widehat{b^{f}}$ : all such strategies with $b^{f} \geq \widehat{b^{f}}$ remain undominated.

In the following proposition, we state conditions under which the supplementary round of a CCA is dominance solvable, and show the equilibrium outcome.

Proposition 3. Let the following conditions hold as public knowledge in the supplementary round:

1. All bidders have bid truthfully in the clock phase;

2. All bidders have been active in the final clock round, where aggregate demand equals supply, i.e., $\sum_{i}\left(\pi_{i}^{f}\right)=\bar{\pi}$;

3. For each bidder $i$, the value $v_{i}^{f}$ for the final clock round package exceeds the KO-bid $\widehat{b_{l}^{f}}$, i.e., $v_{i}^{f}>\widehat{b_{l}^{f}}$;

4. Each bidder $i$ bids only for the final clock round package $\pi_{i}^{f}$ and for packages that require strictly more eligibility points than $\pi_{i}^{f}$, i.e., $e \cdot \pi_{i}^{\alpha}>e \cdot \pi_{i}^{f}$ for any $\pi_{i}^{\alpha} \in \Psi_{i} \backslash\left\{\pi_{i}^{f}\right\}$.

Then, the outcome of the supplementary round is uniquely determined by iterative elimination of weakly dominated strategies. In equilibrium, each bidder bids $b_{i}^{f} \geq \widehat{b_{l}^{f}}$ on package $\pi_{i}^{f}$, and bids the maximum (cap) bids $B_{i}^{\alpha}$ for (some) other packages $\pi_{i}^{\alpha}$. In equilibrium, each bidder gets package $\pi_{i}^{f}$, and pays his KO-bid $\widehat{b_{l}^{f}}$.

The term 'some' in Proposition 3 needs a further explanation. In computing the VCG price that bidder $j$ pays for package $\pi_{j}^{f}$, some bids $b_{i}^{\alpha}$ for some packages $\pi_{i}^{\alpha}$ may be redundant. In 
such a case, iterative elimination of weakly dominated strategies does not specify how high these bids should be; these bids are not actively used in the determination of the winning allocation (i.e., they are not allocation relevant), nor are they used in the VCG pricing (i.e., they are not price relevant either).

There are two important conditions in Proposition 3, conditions 3 and 4. In quite a few auctions in Europe (such as Switzerland and The Netherlands in 2012, Ireland and Austria in 2013), where the main spectrum holdings of incumbent operators were resold in the auction, bidders need to obtain a minimum package size to be able to serve their existing clients. For smaller packages, bidders may have an intrinsic valuation that is relatively low. Moreover, depending on the specific rules related to spectrum caps, it may be unlikely that allocation relevant or price relevant bids on packages that are smaller ${ }^{15}$ than the final clock round package are raising rivals' cost. In these circumstances, it may not be unreasonable to assume bidders do not bid on such smaller packages. ${ }^{16}$ In the next Section, we present an example where allocation relevant or price relevant bids in the supplementary round on packages that are smaller than the last clock round package are dominated so that bidders can predict that other rational bidders will not bid on these strategies. As it is difficult to specify general conditions when relevant bids on smaller packages are dominated, we have formulated condition 4 in the way it is formulated in Proposition 3.

In auctions where all current spectrum holdings are for sale, the value $v^{f}$ equals the company value for incumbents (as without the licenses an incumbent could not continue to operate), which typically is a (very) large number. Depending on the development of the clock phase, it is not unreasonable to assume (condition 3) that in fact $\widehat{b^{f}}<v^{f}$. We have assumed that the values are privately known. The common knowledge of $\widehat{b^{f}}<v^{f}$ implies then that all bidders know that the lower bound on a company's valuation is larger than the KO-bid.

${ }^{15}$ We refer to the "size" of a package as the number of eligibility points it requires.

16 The Austrian regulator RTR confirms that relatively few bids on smaller packages were made and that the price limits for smaller packages were at times only utilized to the extent of between $60 \%$ and 70\%; see https://www.rtr.at/en/pr/PI28102013TK. 
When bidders also bid on packages that are smaller than their final clock round package (note that because of the auction rules, they could never have bid on such packages in the clock phase), the supplementary round is not dominance solvable. Thus, it is not necessarily true that in equilibrium, bidders bid the maximum caps. The danger of doing so is that such bids may become winning bids in combination with bids of others on smaller packages. In that case bidders may be reluctant to express high bids on larger packages with the sole purpose of raising rivals' cost and lower auction prices result. ${ }^{17}$

Again, it is important to point at the crucial difference between a CCA and a VCG mechanism in the presence of preferences for raising rivals' cost: the VCG mechanism is never dominance solvable. It is still true that bidding below value is dominated. However, bidding above value is not dominated under VCG. Bidding above value may result in negative surplus, but it may also result in positive surplus with competitors paying more. The restrictions imposed by the CCA give certainty that bidding above marginal value on larger packages than the final clock round package is without risk of winning these packages if bidders do not choose weakly dominated strategies, and bidders know that others do not bid on smaller packages. Thus, under some conditions, information from the clock phase can be used to raise rivals' cost. This makes the CCA quite different from the VCG mechanism, where raising rivals’ cost is much more risky as bids are unconstrained.

\subsection{The Clock Phase}

We now consider whether it is optimal to bid truthfully in the clock round. To see the consequences of the behavior in the supplementary round for bidding behavior in the clock phase, let us consider the final clock round in which one bidder $i$ has switched from package

17 This discussion may point at an explanation for why auction price in the 2013 UK auction were considered to be on the low side, whereas prices in the 2012 Irish and Dutch auctions were much higher than expected. In Ireland and the Netherlands, incumbents needed to obtain a minimum amount of spectrum to guarantee service to their current customers. Bidding on (very) small packages was not really an option. Moreover, values were close to the overall value of the company. In the UK, on the contrary, only "new" spectrum was auctioned, and there was much less of a need to obtain at least a minimal amount of spectrum. Nevertheless, even in the UK, only 30 bids (out of 277 supplementary round bids) were for smaller packages. 
$\pi^{\alpha}=d_{i}^{T-1}$ to his final package $\pi^{f}=d_{i}^{T}$ thereby ending the auction. In the following proposition, we show that if the KO-bids remain constant and are below values, such behavior of bidder $i$ is strictly suboptimal, and he has an incentive to keep bidding for $\pi^{\alpha}$ and not to switch to $\pi^{f}$ in round $T$.

Proposition 4. Let conditions 2 - 4 of Proposition 3 hold. Suppose that in the final clock round $T$, bidder $i$ has switched from package $\pi_{i}^{\alpha}=d_{i}^{T-1}$ to his final package $\pi_{i}^{f}=d_{i}^{T}$, $\pi_{i}^{f} \neq \pi_{i}^{\alpha}$. Then, this switching from $\pi_{i}^{\alpha}$ to $\pi_{i}^{f}$ in round $T$ is dominated by continuing bidding for package $\pi_{i}^{\alpha}$ in round $T$ and switching to $\pi_{i}^{f}$ in round $(T+1)$ ending the clock phase if the following two conditions are met:

1. All other bidders keep bidding for the same packages in rounds $T$ and $(T+1)$, i.e., $d_{j}^{T+1}=d_{j}^{T}=\pi_{j}^{f}$ for all $j \neq i$.

2. The KO-bid of each bidder $i$ is only determined by bids of other bidders in the supplementary round for packages that require more eligibility points than $\pi_{j}^{f}$.

Proposition 4 basically says that if other bidders do not change their bids if the clock phase lasts one more round, then the bidder under consideration prefers to prolong the clock phase by one round. In doing so, he relaxes the caps on bid increments on his own non-winning bids, without affecting the final allocation of packages. In this way, he can increase the payment of others without affecting his own payments. At the same time, the caps of the other bidders for packages that are larger than the last clock round packages (these are the packages determining his KO-bid) remain intact, and so does the auction price he pays.

Proposition 4 points out that truthful (or sincere) bidding, i.e., bidding according to value is not a dominant strategy in the clock phase. Optimal behavior in the clock phase depends on the behavior of others, and there is a serious motive to prolong the clock phase in order to be able to raise rivals' cost to the maximum extent. If all bidders behave this way, final payments may be considerably larger than when all bidders would bid straightforwardly. 
Section 6 in Levin and Skrzypacz (2013) indicates that when both bidders have lexicographic preferences and unrestricted strategies, they engage in demand reduction in the clock phase. Our Proposition 4 suggests that players engage in demand expansion even in the clock phase. Their analysis rests on the assumption that the there are no strong complementarities and that the value of a small (demand reduction) package is relatively high. In that case, condition 1 may be violated as bidders will respond to the others' intension to expand demand. In the next Section, we show in an example with a multi-commodity CCA that condition 1 may well hold when bidders do not value small packages. In that case, bidders engage in demand expansion.

Together, propositions 1-4 show the potential for the first gaming aspect alluded to in the Introduction: it is optimal not to bid value in a CCA, and bidders are able to raise rivals' cost. The propositions do not intend to claim it is easy to bid in a CCA; they are true under restrictive assumptions, and the question is whether bidders are willing to make these same assumptions. That requires both judgment and gaming, issues we further discuss in Section 6.

\section{An Example}

In this section, we present an example illustrating the mechanism of the CCA and the propositions we have stated in Section 3. In general terms, it is difficult to state how large some of the effects of gaming on the auction outcome can be. This section shows, by means of an example, that the effects can be very substantial. We also show what can happen if some of the assumptions of the Propositions do not hold.

Our example has three (ex ante identical) bidders, two bands, and three blocks that are available in each of the bands. The reserve prices are 1 for blocks in band 1 , and 4 for blocks in band 2. We assume throughout that the auction rules restrict individual bidders to bid for no more than tree licenses in total. Intrinsic values and eligibility points are given in Table 


\begin{tabular}{|c|c|c|c|}
\hline Package no. & Package & Package eligibility & Package value \\
\hline 1 & $(1,2)$ & 21 & 50 \\
\hline 2 & $(2,1)$ & 18 & 46.5 \\
\hline 3 & $(1,1)$ & 13 & 40 \\
\hline
\end{tabular}

Table 1. Values and eligibility points for the three packages.

$1^{18}$ Bidders are intrinsically interested in three packages $(x, y)$, where $x$ and $y$ stand for the numbers of blocks in bands 1 and 2 correspondingly. Blocks in band 1 and 2 "cost” 5 and 8 eligibility points, respectively. As before, bidders have lexicographic preferences for raising competitors’ payments.

We first illustrate bidding in the supplementary round in case bidders have bid straightforwardly in the clock phase. The bid behavior of all three bidders in the clock phase is then as represented in Table 2. Bidders start bidding on package $(1,2)$ as this is the most profitable package: with a value of 50 and a cost of 9 at the reserve prices, the net surplus is 41. As there is excess demand in band 2 (total demand is 6 as all bidders demand 2 blocks), the band 2 price increases. This is not the case in band 1 as total demand (of 3 as each bidder demands 1 block) is smaller than or equal to supply (3 blocks). In round 2, they switch to package $(2,1)$ and then prices increase for block 1 only. When prices reach $(7,5)$ in round 11 , bidders reduce demand to package $(1,1)$ and the clock phase is over.

The highest total bids the players made for the three respective packages are 9,17 , and 12. The relative cap, as explained in Section 2, now works as follows. For package $(1,1)$ bidders are unconstrained. Suppose they bid $b^{(1,1)}$ for $(1,1)$. For their supplementary bid on package $(2,1)$, the final round is the anchor round, and at these prices package $(2,1)$ costs $7 \cdot(2-1)+5 \cdot(1-1)=7$ more than package $(1,1)$. Therefore, bidders can bid for package $(2,1)$ up to $B^{(2,1)}=b^{(1,1)}+7$. For package $(1,2)$, round 2 is the anchor round, and at these prices package $(1,2)$ costs $1 \cdot(1-2)+5 \cdot(2-1)=4$ more than package $(2,1)$.

18 The example captures important elements of telecom auctions. The number of bidders is limited (usually, only incumbents, but sometimes there is a limited number of entrants as well). Incumbents (especially if they have similar market shares) often have similar values, and they expect others to make similar calculations. Finally, the set of spectrum bands available is also very limited. 


\begin{tabular}{|c|c|c|c|c|c|c|c|}
\hline \multirow{2}{*}{$\begin{array}{c}\text { Round } \\
\text { no. }\end{array}$} & \multirow{2}{*}{ Prices } & \multicolumn{4}{|c|}{ Package costs, values, and surplus } & \multirow{2}{*}{$\begin{array}{l}\text { Optimal } \\
\text { package }\end{array}$} & \multirow{2}{*}{ Eligibility } \\
\hline & & Package & Cost & Value & Surplus & & \\
\hline \multirow{3}{*}{1} & \multirow{3}{*}{$(1,4)$} & $(1,2)$ & 9 & 50 & 41 & \multirow{3}{*}{$(1,2)$} & \multirow{3}{*}{21} \\
\hline & & $(2,1)$ & 6 & 46.5 & 40.5 & & \\
\hline & & $(1,1)$ & 5 & 40 & 35 & & \\
\hline \multirow{3}{*}{2} & \multirow{3}{*}{$(1,5)$} & $(1,2)$ & 11 & 50 & 39 & \multirow{3}{*}{$(2,1)$} & \multirow{3}{*}{18} \\
\hline & & $(2,1)$ & 7 & 46.5 & 39.5 & & \\
\hline & & $(1,1)$ & 6 & 40 & 34 & & \\
\hline \multirow{2}{*}{3} & \multirow{2}{*}{$(2,5)$} & $(2,1)$ & 9 & 46.5 & 37.5 & \multirow{2}{*}{$(2,1)$} & \multirow{2}{*}{18} \\
\hline & & $(1,1)$ & 7 & 40 & 33 & & \\
\hline \multirow{2}{*}{4} & \multirow{2}{*}{$(3,5)$} & $(2,1)$ & 11 & 46.5 & 35.5 & \multirow{2}{*}{$(2,1)$} & \multirow{2}{*}{18} \\
\hline & & $(1,1)$ & 8 & 40 & 32 & & \\
\hline \multirow{2}{*}{5} & \multirow{2}{*}{$(4,5)$} & $(2,1)$ & 13 & 46.5 & 33.5 & \multirow{2}{*}{$(2,1)$} & \multirow{2}{*}{18} \\
\hline & & $(1,1)$ & 9 & 40 & 31 & & \\
\hline \multirow{2}{*}{6} & \multirow{2}{*}{$(5,5)$} & $(2,1)$ & 15 & 46.5 & 31.5 & \multirow{2}{*}{$(2,1)$} & \multirow{2}{*}{18} \\
\hline & & $(1,1)$ & 10 & 40 & 30 & & \\
\hline \multirow{2}{*}{7} & \multirow{2}{*}{$(6,5)$} & $(2,1)$ & 17 & 46.5 & 29.5 & \multirow{2}{*}{$(2,1)$} & \multirow{2}{*}{18} \\
\hline & & $(1,1)$ & 11 & 40 & 29 & & \\
\hline \multirow{2}{*}{8} & \multirow{2}{*}{$(7,5)$} & $(2,1)$ & 19 & 46.5 & 27.5 & (11) & 1 \\
\hline & & $(1,1)$ & 12 & 40 & 28 & $(1,1)$ & 13 \\
\hline
\end{tabular}

Table 2. Clock phase development.

Therefore, bidders can bid for package $(1,2)$ up to $B^{(1,2)}=b^{(2,1)}+4$, where $b^{(2,1)} \leq B^{(2,1)}$ is the actual highest bid for package $(2,1)$ in the clock phase or the supplementary round.

In addition, bidders could also make bids on packages $(3,0)$ and $(0,3)$ in the supplementary round, and on smaller packages than $(1,1)$. Bidders do not have an intrinsic value for these packages, but if bidding on these packages increases the prices to be paid by competitors (without obtaining them), then it may be optimal to also express bids for these packages. For package $(3,0)$, the anchor round is the final clock round, and at these prices package $(3,0)$ costs $7 \cdot(3-1)+5 \cdot(0-1)=9$ more than package $(1,1)$. Therefore, bidders can bid for package $(3,0)$ up to $B^{(3,0)}=b^{(1,1)}+9$.

For package $(0,3)$, the anchor round is the first clock round, and at these prices package $(0,3)$ costs $1 \cdot(0-1)+4 \cdot(3-2)=3$ more than package $(1,2)$. Therefore, bidders can bid for package $(0,3)$ up to $B^{(0,3)}=b^{(1,2)}+3$, where $b^{(1,2)} \leq B^{(1,2)}$ is the actual bid for 
package $(1,2)$ in the clock phase or in the supplementary round. The impact of bidding on smaller packages will be discussed at a later point in this section. ${ }^{19}$

As a reference point on auction prices, we provide two benchmarks. The first is the prices that would result if all bidders bid their intrinsic values. It is easy to see that each bidder imposes an opportunity cost of 16.5 on the two competitors. These opportunity costs imposed upon others could be considered as a kind of natural market price for obtaining the package $(1,1)$. As a second benchmark, if bidders do not make additional bids in the supplementary phase, each of them obtains package $(1,1)$ and pays $(17+12)-$ $(12+12)=5$. Here, $(12+12)$ is the sum of bidder $i$ 's competitors' winning bids, and $(17+12)$ is the total sum of winning bids if bidder $i$ were not present (two remaining bidders would have obtained packages $(2,1)$ and $(1,1))$. It is easy to see that VCG prices in this example are in the core, so that the core selection requirements that CCA imposes on prices are not binding.

\subsection{The Supplementary Phase}

Now that we have illustrated how the relative cap works in our example and how the price determination rule works, we want to illustrate our Propositions and to show how large the effect due to the lexicographic preference for raising rivals' cost can be in a CCA. In the supplementary round, players' bids can be expressed as $\left(b^{(1,1)}, b^{(2,1)}, b^{(1,2)}, b^{(3,0)}, b^{(0,3)}\right)=$ $(b, b+x, b+x+y, b+z, b+x+y+t)$. As argued above, the relative cap implies that given the behavior in the clock phase reported in Table 2, $x \leq 7, y \leq 4, z \leq 9$, and $t \leq 3$. This implies that the maximal sum of bids of a two-winner combination is $2 b+23$, which is a combination of $b^{(3,0)}$ and $b^{(0,3)}: b^{(3,0)}+b^{(0,3)}=2 b+z+x+y+t \leq 2 b+23$.

In order to guarantee that the bid on the final clock round package $(1,1)$ is the winning bid, a bidder should bid at least $b=23$, which is the KO-bid. If others truly believe that

${ }^{19}$ Although the package $(0,2)$ is not smaller than $(1,1)$, it does not play much of a role in a full analysis of the example. For didactical purposes, we do not take bids on this package into account in our discussion. 
competitors are bidding $b \geq 23$ on package $(1,1)$, they find it optimal to bid $(23,34,30,32,37)$ on the five possible packages in order to win $(1,1)$ themselves, and to make sure that competitors are paying the maximal amounts for their winning bid.

The statement below captures this reasoning. This statement illustrates Propositions 1-3 and follows from iterative elimination of weakly dominated strategies. In a first elimination round, bidders bid $b \geq 23$ on $(1,1)$ to guarantee they are among the winners and bid their marginal valuations in addition to $b$ on packages $(2,1)$ and $(1,2)$. It is clear that this KO-bid $\widehat{b^{f}}=23$ is smaller than valuation $v=40$. In the second elimination round, when bidders bid at least $\widehat{b^{f}}=23$ on package $(1,1)$, the preference for raising rivals' cost ranks all the undominated strategies with respect to the price that others pay. Thus, the only strategies surviving the second round of elimination are such that bidders bid the largest amounts that are consistent with the relative cap on all packages that have an influence on competitor's prices.

Statement 1. Let bidders bid truthfully in the clock phase. Then, if it is publicly known that bidders only bid for packages $(1,1),(2,1),(1,2),(3,0),(0,3)$, then only strategies of the form $(b, b+7, b+11, b+9, b+14)$ with $b \geq 23$ survive iterative elimination of weakly dominated strategies. In the resulting equilibrium, each bidder gets package $(1,1)$ and pays auction price of 23 .

One may wonder whether it is risky to bid (so high) on packages that do not have intrinsic value, such as $(3,0)$ and $(0,3)$. The answer depends on whether bidders are reasonably certain about the competitors' values and their rationality, and on whether bidders are ready to assume that others do not bid on smaller packages. If rivals expect others may place a (high) bid on packages $(3,0)$ and $(0,3)$, then they want to protect themselves against not winning, calculate the KO-bid, and bid this amount. In this case, there is no risk for rivals to place (high) bids on $(3,0)$ and $(0,3)$, but this uncertainty is endogenous to the auction in that others' bids depend on their expectations of what others' bid. Of course, if one of the bidders 
does not understand the iterative dominance logic, there may be two bidders winning a package they do not value.

When clock phase bids of individual bidders are not public information, bidders cannot compute others' relative caps and, therefore, their own KO-bids. As explained in the previous section, when only total demands in each clock round are announced, each bidder can calculate for any possible development of the clock phase the corresponding KO-bid. The maximum of all such bids is the true KO-bid which guarantees the bidder winning his final clock round package irrespective of the clock phase development. Given that bidders cannot bid for more than 3 licenses, there are 14 generically different scenarios that generate the same joint demand of bidders 2 and 3. Computing KO-bids for each scenario yields a maximum KO-bid of 29. This implies that in order to win, bidder 1 has to bid $b \geq 29$. $^{20}$

The example illustrates that the lexicographic preference for raising rivals' cost can have devastating effects for the bidders. Instead of paying the VCG prices of 16.5 for package $(1,1)$, which would have resulted had they bid values on all packages, they are now ending up with the same winning allocation, but having to pay 23!

What happens if bidders also consider making supplementary bids on smaller packages? The final clock round is the anchor round for these smaller packages and it is easy to see that bidders can maximally make the following bids:

$$
b^{(1,0)} \leq(b-5), b^{(0,1)} \leq(b-7) \text {, and } b^{(2,0)} \leq(b+2) .
$$

If every bidder bids maximally on all eight possible packages, including these tree smaller ones, then the winning allocation will be $(0,3),(1,0)$, and $(2,0)$ as the total bid on this combination is $(3 b+11)$ and prices will be 26,7 and 14 correspondingly. Bidding high on packages that are larger than the final clock round package may seem to be risky then.

We will argue now, however, that if bidders have sufficiently low intrinsic value for packages smaller than $(1,1)$, any combination of bids that survives iterative elimination of

\footnotetext{
${ }^{20}$ The details of these derivations are in Appendix III.
} 
dominated strategies, does not have bids on packages $(1,0)$ and $(2,0)$ that are either pay-off or allocation relevant. ${ }^{21}$

In order to see this we first consider the question whether bids on packages $(1,0)$ and $(2,0)$ that are allocation irrelevant could potentially be price relevant, and whether bidders would like to make such bids as a part of a combination of bids in the supplementary round. If this were so, bidders could safely bid on these packages without winning them. The answer to this question is negative. Indeed, these bids can only be price relevant if they form the highest total value in conjunction with a bid of one other player. Given that players can only bid for three licenses at most (and that there are three units of each license), it must be that the best combination of bids is with the other bidder's bid for $(0,3)$. As the other bidder can bid at most $B^{(0,3)}=b^{(1,2)}+3$, the total bid on the two packages cannot be larger than $b^{(1,2)}+$ $3+(b-5)$ if in combination with the bid for $(1,0)$, or $b^{(1,2)}+3+(b+2)$ if in combination with the bid for $(2,0)$.

However, instead of (or, in addition to) making a price relevant bid on either $(1,0)$ or $(2,0)$ the bidder can also make a combination of bids that includes a bid of $(b+6)$ on package $(2,1){ }^{22}$ Such a bid can be combined with the rival bid on $(1,2)$ to make a possible joint bid of $b^{(1,2)}+(b+6)$. As this is larger than both expressions given before, combination of bids that includes price relevant bids on $(1,0)$ and $(2,0)$ are dominated by other bids. ${ }^{23}$ Moreover, it is clear that if the intrinsic valuations are low enough, bids on packages $(1,0)$ and $(2,0)$ that are allocation relevant are weakly dominated as the minimum price for each unit is the (positive) reserve price. A similar argument holds true for packages $(0,1)$ and $(0,2)$.

${ }^{21}$ We are not concerned with bids on some packages that are neither allocation nor price relevant. It is clear that there will be many bids that are equivalent in terms of allocation and prices. For example, a low bid on $(1,0)$ may not be dominated, but is also completely irrelevant.

${ }^{22}$ As the bidder bids less than his marginal valuation on $(2,1)$ compared to the bid on $(1,1)$, he will not regret if he wins that package instead of $(1,1)$.

${ }^{23}$ Note also that if a bid on a package is allocation or price irrelevant given a larger set of possible strategies opponents choose, these bids are also irrelevant in a smaller set of possible strategies. Thus, if these strategies are not considered relevant in an early stage of the iterative elimination process of dominated strategies, then they are also irrelevant at a later stage. 
Thus, in this example there is a good reason why bidders do not consider bidding on smaller packages. Bidders can safely bid on larger packages if they believe that other bidders will not make dominated supplementary bids.

\subsection{The Clock Phase}

We have argued in Section 3 that bidders may have an incentive to prolong the clock round of the auction to be more effective in raising rivals' cost in the supplementary phase. Here, we illustrate this effect and show that it may even hold if others' bidding behavior is not kept constant. ${ }^{24}$ To see this, suppose that all bidders continue bidding in the clock phase on $(2,1)$ until the prices reach the amount $(x, 5)$, for some $x$ and then drop demands to $(1,1)$, finishing off the clock phase. This implies that on package $(3,0)$, bidders can pay up to $(2 x-5)$ more than they bid on $(1,1)$ in the supplementary round. The additional amount they can bid on $(0,3)$ is $(x+7)$ so that the total amount they can bid more on the combination $(3,0)$ and $(0,3)$ is $(3 x+2)$. The next statement says that the clock phase cannot end with all bidders bidding for package $(1,1)$ until the moment the continuation bids in the supplementary round are such that all surplus is dissipated.

Statement 2. Let bidders have bid truthfully in the clock phase until clock round 8. Then:

1. If there is an equilibrium of the continuation game in which all bidders switch from $(2,1)$ to $(1,1)$, and the clock phase ends with all bidders bidding for the package $(1,1)$ in the last clock round $T$, then it must be that $T \geq 14$.

2. If there is an equilibrium of the continuation game in which all bidders switch from $(2,1)$ to $(1,1)$ in the last clock round $T=14$, the supplementary round bids are $B=$ $\left(40,53,57, b_{(3,0)}, b_{(0,3)}\right)$, where $b_{(3,0)} \in[60,61]$ and $b_{(0,3)} \in[59,60]$. Winners pay a price of at least 39 .

${ }^{24}$ This requires, of course, that bidders have reasonably accurate expectations about the valuations of other bidders. 
Note that the behavior in the clock phase has a Prisoners' Dilemma aspect: it is individually rational to continue the clock phase when the others already have reduced demands to $(1,1)$, but if everyone acts this way, the bidders collectively get a worse outcome. Note also that the supplementary round strategy suggests bidders to bid in the range $59-60$ on both packages $(3,0)$ and $(0,3)$, which bidders do not intrinsically value. This implies there are multiple equilibria, and that if bidders do not coordinate on the same equilibrium, some bidders may win packages $(3,0)$ and $(0,3) .{ }^{25}$ Strategic bidding results in the clock phase not providing useful information concerning the marginal valuations of bidders.

Note also that there are strong strategic complementarities in this example as bidders do not value packages smaller than $(1,1)$. This implies that bidding on packages smaller than $(1,1)$ is not gainful and bidders will not engage in strategic demand reduction (unlike Levin and Skrzypacz, 2013).

\section{Budget Constraint Bidders}

In Section 4, we have considered how bidders can raise rivals' cost considerably, by including bids on packages they do not intrinsically value and/or by bidding strategically (above marginal value) in the clock phase. This may require, however, that bidders have very large budgets, a condition that is usually not fulfilled. In this section, we analyze some gaming aspects related to budget constraints.

Under budget constraints, some fundamental questions arise as to what it means to have a budget constraint in a CCA, and how to bid with it. To understand the issues involved, reconsider the example we analyzed in Section 4, where all bidders have been active in the clock phase until round 14 , where prices reached $(13,5)$ and then dropped demand from $(2,1)$ to $(1,1)$. Suppose now, however, that bidders have a budget constraint of 35 . Note that the highest bid made in the clock phase is 29 (on package $(2,1)$ at prices $(12,5)$ ), while the final

25 The multiplicity of equilibria originates from the finite bid increment. The smaller is the bid increment, the smaller the range of multiplicity is, and in the limit, when the clock prices increase continuously, there is a unique outcome, which survives iterative elimination of dominated strategies. 
clock phase bid is 18 on package $(1,1)$ so that the budget constraint was not binding in the clock phase.

As bidders know the others could together bid up to (more than) 40 more on the combination of $(3,0)$ and $(0,3)$, they may actually bid in the supplementary round their entire budget of 35 on package $(1,1)$ to maximizes their chances to win some package. What could they bid on other packages without running the risk of having to pay more than 35 ? The answer is that on both packages $(2,1)$ and $(1,2)$ they should not bid more than 40 , otherwise they will have a chance to pay more than their budget.

To see this, consider bids $B=\left(b_{(1,1)}, b_{(2,1)}, b_{(1,2)}\right)$ with $b_{(1,1)}=35$. The relative cap restrictions are $b_{(2,1)} \leq b_{(1,1)}+13$ and $b_{(1,2)} \leq b_{(1,1)}+17$. This implies bidders 2 and 3 can together maximally bid 30 more for the total spectrum than they can together bid for $(2,2)$. Thus, if bidder 1 does not bid more than 5 more for a package $(2,1)$ or $(1,2)$ he does not risk paying above 35. To see that a bid larger than 40 is risky, suppose that bidder 1 bids $B_{1}=$ $(35,40,40+x), x>0$. It is easy to see that if bidder 2 bids $B_{2}=\left(18+\frac{3}{4} x, 29,35+\frac{1}{2} x\right)$ while bidder 3 bids $B_{3}=(22,35,35)$, bidder 1 wins $(1,2)$ - together with bidder 3 winning $(2,1)$ - and pays $p_{(1,2)}=35+\frac{1}{2} x$, i.e., above budget. A similar argument holds true for bidder 1 bidding $(35,40+x, 40), x>0$.

Two things are worth noting about this conclusion. First, if all bidders behave in this way and bid $B=(35,40,40)$, the actual payments will be 10 (!) for the package (1,1), quite a bit lower than the reasonable market prices of 16.5, and much less than 40 (when bidders are not constrained by budgets). Second, a budget constraint of 35 does not mean that a bidder will not express a bid that is larger than 35. A bidder can calculate that bidding 40 on $(2,1)$ and/or $(1,2)$ will never be winning bids, if combined with a bid of 35 on $(1,1)$. Thus, even a hard budget constraint does not imply that you cannot (and should not) make a bid above the budget constraint!

The problem with the behavior assumed above is that it is not an equilibrium. In particular, assuming its rival bidders avoid any risk (of not winning $(1,1)$ package) by bidding 
$B=(35,40,40)$, a bidder may increase rivals’ prices. The next proposition shows that this is a fairly general phenomenon.

Proposition 5. Let each bidder $i$ have a budget $D_{i}$ that is lower than his KO-bid $\widehat{b_{\imath}^{f}}$ in the supplementary round, which is in turn lower than the value $v_{i}^{f}$, i.e., $D_{i}<\widehat{b_{l}^{f}}<v_{i}^{f}$. Let also all bidders be active in the final clock round. Then, in any undominated equilibrium, at least one bidder bids above his budget.

Thus, bidding under a budget constraint is fairly complicated in a CCA where bidders are interested in raising rivals' cost. It is not optimal to have all bids satisfy the budget constraint, and one can "safely"26 make some bids above this constraint. Generally, it is not optimal, however, in a CCA to make only these "safe" bids; it can be optimal to take risks. How far one should go, depends on the expectations of how far others are willing to go and in a oneoff round, this is very difficult to predict.

The next statement provides an example and shows that a CCA may well exhibit many asymmetric equilibria, which all have a Hawk-Dove nature, where the more aggressive, more risk-taking bidder does better than the less aggressive bidder. The statement presents one such an equilibrium.

${ }^{26}$ Of course, what is important here is our assumption that players know the bidding behavior of individual bidders. In real CCAs, bidders are only informed about the total demand per band in each clock round. In that case, bidders can still make inferences about which bids they can safely make, but this requires more complicated combinatorial calculations. 
Statement 3. Let all bidders have dropped demand in clock round 14 at prices $(13,5)$ from $(2,1)$ to $(1,1)$, and let all bidders have a budget of 35 . The following is an equilibrium of the supplementary round: one "Hawk-like" bidder bids $B^{\mathrm{H}}=(35,48,52,56,55)$, while the other two "Dove-like" bidders bid $B^{\mathrm{D}}=(35,48,52,50,49)$. All bidders win package $(1,1)$, the "Hawk-like" bidder pays $p^{\mathrm{H}}=30$, while the other two pay $p^{\mathrm{D}}=35$.

The equilibrium presented in Statement 3 satisfies the budget constraint “in equilibrium”, but all bidders run the risk of having to pay more than their budget if a non-equilibrium allocation results. ${ }^{27}$ This "equilibrium" interpretation of a budget constraint is the third interpretation of a budget constraint (apart from the "never make a bid above the budget constraint" - which is not optimal in a CCA, and "make only bids above the budget constraint that are never winning”).

The equilibrium in Statement 3 is only one of the equilibria of the budget-constrained game. Multiple equilibria create endogenous uncertainty for bidders, as they have no way to coordinate on one of them. If they fail to coordinate, they may have to pay above their budget and obtain (and pay for) a package they do not value. Bidding under a budget constraint is, therefore, a risky endeavor, especially as the most careful bidder is likely to pay the most at the end of the auction.

\section{Discussion and Conclusion}

This paper has considered combinatorial clock auctions (CCAs) where bidders are not only interested in the package they acquire and the price they have to pay, but also care about the price competitors have to pay. We have also analyzed some implications of budget constraints. We have argued that these preferences and constraints are likely to be important in markets where CCAs are nowadays used.

${ }^{27}$ Salant (2013, p. 179) presents an example where a VCG with budget constraint bidders does not have an equilibrium in pure strategies. 
We have shown that given these preferences, CCAs give rise to many strategic gaming possibilities. First, by placing bids on non-winning packages (in the extreme, on packages bidders do not intrinsically value) a CCA gives players the possibility to raise rivals' cost. Information provided in the clock phase, together with the rules capping the bids in the supplementary round, allows bidders to calculate a "knock-out" price, which is the bid that bidders minimally have to make to guarantee themselves winning some package. ${ }^{28}$

We have shown that in many circumstances, all undominated strategies for the supplementary round require bidders to bid at least this "knock-out" amount on the final clock round package. Given this conclusion, it is without risk to bid above value on some other packages to raise rivals' cost. How much to bid above value depends, however, on expectations concerning rivals' behavior. If bidders know that "knock-out" bids of other bidders are within their budgets and below valuations, and if they know (or, can anticipate, as in the example in Section 4) that other bidders are not making bids on smaller packages, then bidders will raise their bids on non-winning packages to the maximal extent possible. This is the unique outcome that survives iterative elimination of dominated strategies.

Second, gaming in the clock phase is possible to soften the constraints imposed by the relative cap for bidding in the supplementary phase. In this way, the clock phase looses much of its appeal as a "price discovery vehicle”. There is an important danger both for the bidders and for auctioneer interested in allocating packages efficiently that if one of the players does not fully understand the scope for gaming, the auction outcome can be very inefficient.

We have also pointed out that it is not clear how to interpret "bidding under a budget constraint" in a CCA. We have shown it is not optimal to place only bids that satisfy the budget constraint. A bidder can calculate that certain bids above budget do not have a chance of winning if properly combined with other bids. These bids may nevertheless be essential in raising rivals' cost. If other bidders are also constrained, a bidder may even make higher bids (much above budget) on certain packages he hopes not to win, as he knows that if others are

${ }^{28}$ In this paper, we have considered the relative cap rule that is often adopted in CCAs. In Appendix II, we argue that similar considerations apply to the final cap rule. 
constrained, he will not win these bids. These bids are, however, risky (and can be winning bids) if others will also make these risky bids. Not making these bids may result, however, in having to pay much more than competitors for similar packages. With budget constraints, CCAs may place bidders in a situation where risky choices are unavoidable, similar to the choices players have to make in a Hawk-Dove game with multiple, asymmetric equilibria.

One may argue that it is too much to require efficient outcomes of any auction format if bidders are budget constraint and spite is a motive for bidding. Dobyinski et al. (2012), e.g., show that when bidders have a budget constraint, there is no deterministic multi-unit auction that (i) is individually rational and dominant-strategy incentive-compatible, (ii) makes no positive transfers, and (iii) always produces a Pareto optimal outcome. It is thus not surprising that the CCA does not always yield efficient outcomes. Our results, however, point in the direction that if bidders can use bids on non-winning packages to raise rivals' cost, the one off supplementary round creates so much uncertainty for bidders (e.g., because of the multiplicity of equilibria) that auction outcomes may be far off from the Pareto frontier.

One problem with CCAs is that given our current knowledge, it is far from clear what the optimal bidding strategy for bidders is. Our results can only characterize the optimal strategies under restrictive assumptions and it is a matter of judgment whether bidders believe in these assumptions about rival bidders. One of these assumptions relates to the motives of other bidders. Another relates to the question whether or not demand equals supply in the final clock round. To better understand the inefficiencies to which CCA potentially gives rise, one should better understand the bidding behavior when our assumptions do not hold.

There are also some issues related to the auction design itself. First, there is the issue of how much information bidders should get in the clock phase, and whether or not there should be a clock phase. Without the clock phase, a CCA reduces to a VCG mechanism, with a different, core-selecting pricing rule. ${ }^{29}$ If there is a clock phase, bidders could, in principle, at the end of each round of the clock phase get information on anonymous individual demands,

${ }^{29}$ We did not consider core selecting pricing rules, and focused on cases where the CCA allocation and prices are in the core, as Goeree and Lien (2012) already point at the fact that bidding valuation is not optimal under alternative pricing rules. 
on total demand only, or only on the fact whether or not in a band demand is larger than supply. In this paper, we have pointed at gaming possibilities that arise when bidders receive some information during the clock phase: either information about individual demands or total demand. In the Austrian 2010 CCA auction, bidders did not get any information in the clock phase apart from the fact that in certain rounds the prices on certain bands increased, implying that demand was higher than supply in these bands. The Austrian model has, however, an important drawback in case bidders have a preference ordering over the full auction outcome (as in the case where the auction outcome partially determines the market structure after the auction and thereby the profits firms make; see footnote 4). It is important to understand the pros and cons of different information structures better. This is an area where future research is necessary.

A second issue relates to the pricing rule. With auction prices that are determined by competitors' bids, and with the possibility of making bids on many different packages, the CCA lends itself to bidders raising rivals’ cost. In 2012, Romania had a package auction, but the second-price principle was replaced by a first price principle (see Ancom, 2012), and goods being allocated at the end of the clock phase (and only unsold goods being offered in the second round). In a first-price auction, one can only raise rivals' cost by also raising the cost for oneself. To better understand the advantages and disadvantages of the second-price principle in package auctions is another area for more research.

A third issue relates to the type of packages bidders may create. It may be useful to see the advantages and drawbacks of allowing bidders to bid for the full spectrum of packages or whether it is better to restrict the number of packages in advance of the auction to make the set of possible outcomes more transparent.

\section{References}

Ancom (2012). Terms of reference for the organization of the competitive selection procedure in view of awarding the rights to use the radio frequencies in the $800 \mathrm{MHz}$, 900 MHz, 1800 MHz and 2600 MHz bands, June 2012. 
Ausubel, L., and P. Cramton (2011). Activity Rules for the Combinatorial Clock Auction, working paper University of Maryland.

Ausubel, L. M., P. Cramton, and P. Milgrom (2006). The Clock-Proxy Auction: A Practical Combinatorial Auction Design, in P. Cramton, Y. Shoham, and R. Steinberg (eds.), Combinatorial Auctions, Chapter 5, 115-138, MIT Press.

Ausubel, L., and P. Milgrom (2002). Ascending Auctions with Package Bidding, Frontiers of Theoretical Economics 1, Article 1.

BAKOM (2011). Ausschreibung von Frequenzblocke für die landesweit Erbringung von mobile Fernmeldediensten in der Schweiz, Anhang III: Auktionsregeln für die kombinierte Vergabe von Frequenzspektrum in den $800 \mathrm{MHz}, 900 \mathrm{MHz}, 1.8 \mathrm{GHz}$, 2.1 GHz und 2.6 GHz-Bändern.

BAKOM (2012). Neue Mobilfunkfrequenzen für Orange, Sunrise und Swisscom, available at: http://www.bakom.admin.ch/themen/frequenzen/03569/index.html?lang=de.

Beck, M., and M. Ott (2011). On Bayesian Equilibrium in Minimum-Revenue CoreSelecting Auctions, preliminary draft available from M. Beck and M. Ott.

Bichler, M., P. Shabalin, and·J. Wolf (2013). Do core-selecting Combinatorial Clock Auctions always lead to high efficiency? An experimental analysis of spectrum auction designs. Experimental Economics, Forthcoming.

Commission for Communications Regulation ComReg (2011). Multi-band Spectrum Release, Draft Information Memorandum, Document No: 11/75, Date: 24 Oct 2011.

Commission for Communications Regulation ComReg (2012). Multi-band Spectrum Release, Release of the $800 \mathrm{MHz}, 900 \mathrm{MHz}$, and $1800 \mathrm{MHz}$ Radio Spectrum Bands, DocumentNo: 12/25, Date: 16 March 2012.

Cooper, D. and H. Fang (2008). Understanding overbidding in second price auctions, an experimental study, The Economic Journal 118, 1572-1595.

Cramton, P. (2012). Spectrum Auction Design, working paper.

Das Varma, G. (2003). Bidding for a process innovation under alternative modes of competition, International Journal of Industrial Organization 21, 15-37.

Day, R.W., and S. Raghavan (2007). Fair Payments for Efficient Allocations in Public Sector Combinatorial Auctions, Management Science 53, 1389-1406.

Day, R.W., and P. Milgrom (2008). Core-selecting Package Auctions, International Journal of Game Theory 36, 393-407. 
Dobzinski, S., R. Lavi, and N. Nisan (2012). Multi-unit auctions with budget limits. Games and Economic Behavior 74, pp. 486-503.

DotEcon (2010). Award of liberalized spectrum in the $900 \mathrm{MHz}$ and otherbands, A report for ComReg, ComReg Document 10/71a.

DotEcon (2012). Issues related to the Draft Information Memorandum. A report for ComReg, ComReg document 12/51, 25 May 2012.

Erdil, A., and P. Klemperer (2009). A new payment rule for core-selecting auctions, Journal of the European Economic Association 8, 537-547.

Fiat, A., A. Karlin, E. Koutsoupias, and A. Vidali (2012). Approaching Utopia: strong truthfulness and externality-resistant mechanisms, at http://arxiv.org/abs/1208.3939v1.

Goeree J.K., and Y. Lien (2009). On the impossibility of core-selecting auctions, Theoretical Economics (forthcoming).

Goeree, J. (2003). Bidding for the future, Journal of Economic Theory 108, 345-64.

Grim, V., F. Riedel, and E. Wolfstetter (2003). Low price equilibrium in multi-unit auctions: the GSM spectrum auction in Germany, International Journal of Industrial Organization 21, 1557-69.

Hoppe, H., P. Jehiel, and B. Moldovanu (2006). License auctions and market structure, Journal of Economics and Management Strategy 15, 371-96.

Janssen, M.C.W., and V. Karamychev (2007). Selection effects in auctions for monopoly rights, Journal of Economic Theory 134, 576-82.

Janssen, M.C.W., and V. Karamychev (2010). Do Auctions select Efficient Firms, Economic Journal 120, 1319-1344.

Janssen, M.C.W., and V. Karamychev (2013). Gaming in the Combinatorial Clock Auction. Tinbergen Institute Discussion Papers, 13-027.

Jehiel, P., and B. Moldovanu (2000). Auctions with downstream interaction among buyers, Rand Journal of Economics 31, 768-91.

Jehiel, P., and B. Moldovanu (2003). An economic perspective on auctions, Economic Policy 36, 271-308.

Jehiel, P., B. Moldovanu, and E. Stacchetti (1996). How (not) to sell nuclear weapons, American Economic Review 86, 814-29.

Klemperer, P. (2002a). What really matters in auction design, Journal of Economic Perspectives 16, 169-89. 
Klemperer, P. (2002b). How not to run auctions: the European 3G mobile telecom auctions, European Economic Review 46, 829-45.

Knapek, S., and A. Wambach (2012). Strategic Complexities in the Combinatorial Clock Auction, CESifo working paper \#3983.

Levin, J., and A. Skrzypacz (2013). Properties of the Combinatorial Clock Auction, Stanford University Working Paper.

Lu, J. (2012). Optimal Auctions with asymmetric financial externalities, Games and Economic Behavior 74, 561-75.

Maasland, E., and S. Onderstal (2007). Auctions wit financial externalities, Economic Theory 32, 551-74.

Morgan, J., K. Steiglitz, and G. Reis (2003). The spite motive and equilibrium bidding in auctions, The B.E. Journal of Economic Analysis \& Policy 2(1) article 5.

Ofcom (2012). Assessment of future mobile competition and award of $800 \mathrm{MHz}$ and 2.6 GHz, July 2012.

Salant, D. (2013). A Primer on Auction Design, Management and Strategy, mimeo, Toulouse School of Economics.

Sharma, A., and T. Sandholm (2010). Asymmetric Spite in Auctions, In Proceedings of the National Joint Conference on Artificial Intelligence (AAAI).

\section{Appendix I}

\section{Proof of Proposition 1.}

For the final cap rule, the proof is in Ausubel and Cramton (2011), propositions 1 and 2. We provide a proof for the relative cap rule used here. Suppose bidder $i$ bids $b_{i}^{f}$ on $\pi_{i}^{f}$ and zero on all other packages. We also assume for a moment that the clock round bids of bidder $i$ are not taking part in computing the optimal allocation. We first show that there is an amount $\widehat{b_{l}^{f}}$ such that if $b_{i}^{f}>\widehat{b_{l}^{f}}$, bidder $i$ necessarily wins $\pi_{i}^{f}$ and pays at most $\widehat{b_{l}^{f}}$. Then, we argue that if we take into account clock phase bids of bidder $i$, and allow him to bid on all packages in the supplementary round, he cannot become a losing bidder: either he is still a winner of $\pi_{i}^{f}$ at the same price $p_{i}^{f}$, or he wins another package, which finishes the proof of the proposition. 
We use the following notation. Let bidder $i$ win package $\pi_{i}$. We denote the maximum of the sum of bids of all other bidders for an allocation $\left\{\pi_{i}^{\alpha}\right\}$ of the remaining $\left(\bar{\pi}-\pi_{i}\right)$ blocks (objects) by $V_{-i}\left(\pi_{i}\right)$, i.e.,:

$$
V_{-i}\left(\pi_{i}\right) \equiv \max _{\left\{\pi_{j}^{\alpha}\right\}: \sum_{j} \pi_{j}^{\alpha} \leq\left(\bar{\pi}-\pi_{i}\right)} \sum_{j} b_{j}^{\alpha} .
$$

If bidder $i$ does not win, we formally set $\pi_{i}=0$ in the above definition. In other words, $V_{-i}(0)$ is the maximum sum of the bids of all other bidders for an allocation of the aggregate supply had bidder $i$ not been present. Since, according to our assumption, bidder $i$ has only one bid $b_{i}^{f}$ on package $\pi_{i}^{f}$, he either wins $\pi_{i}^{f}$ and pays the VCG price

$$
p_{i}^{f}=V_{-i}(0)-V_{-i}\left(\pi_{i}^{f}\right),
$$

or he does not win any package. If he wins $\pi_{i}^{f}$, it must be the case that $b_{i}^{f}+V_{-i}\left(\pi_{i}^{f}\right) \geq$ $V_{-i}(0)$, i.e., $b_{i}^{f} \geq p_{i}^{f}$. What remains to be shown is that $p_{i}^{f}$, which generally depends on all bids of all other bidders, but not on bids of bidder $i$, has an upper-bound which we denote by $\widehat{b_{\imath}^{f}}$. By construction, $\widehat{b_{\imath}^{f}}$ is the highest price for the final clock round package $\pi_{i}^{f}$ that might occur:

$$
\widehat{b_{l}^{f}}=\sup _{\left\{b_{j}^{\alpha}\right\}: \pi_{j}^{\alpha} \in \Pi, j \neq i} p_{i}^{f}=\sup _{\left\{b_{j}^{\alpha}\right\}: \pi_{j}^{\alpha} \in \Pi, j \neq i}\left(V_{-i}(0)-V_{-i}\left(\pi_{i}^{f}\right)\right),
$$

where the maximum is taken over all feasible (satisfying the relative cap rule) bids $b_{i}^{\alpha}$ for all feasible packages $\pi_{i}^{\alpha}$ of all other bidders $j \neq i$. We show now that $\widehat{b_{\imath}^{f}}$ is finite.

According to the relative cap rule, the bid for any package $\pi_{j}^{\alpha}$ that bidder $j$ can make in the supplementary round is capped by a certain amount relative to the bid for package $\pi_{j}^{\beta}$, where $\beta$ is the package that bidder $j$ bid for in the anchor (for package $\pi_{j}^{\alpha}$ ) round, i.e., $b_{j}^{\alpha} \leq b_{j}^{\beta}+c_{j}^{\alpha}$. Continue this process recursively, we write $b_{j}^{\beta} \leq b_{j}^{\gamma}+c_{j}^{\beta}$ and so on, until we reach the final clock round package $\pi_{j}^{f}$. Thus, the relative cap rule implies $b_{j}^{\alpha} \leq b_{j}^{f}+\bar{c}_{j}^{\alpha}$, where the caps $\bar{c}_{j}^{\alpha}$ only depend on the clock phase development. Now we can evaluate $\widehat{b_{l}^{f}}$. 
First, as $\sum_{j} \pi_{j}^{f} \leq\left(\bar{\pi}-\pi_{i}^{f}\right)$, i.e., the allocation of packages $\pi_{j}^{f}$ to all other bidders, is always feasible in the computation of $V_{-i}\left(\pi_{i}^{f}\right)$, it follows that

$$
V_{-i}\left(\pi_{i}^{f}\right) \geq \sum_{j \neq i} b_{j}^{f} .
$$

Using $b_{j}^{\alpha} \leq b_{j}^{f}+\bar{c}_{j}^{\alpha}$, it follows that

$$
\begin{aligned}
& V_{-i}(0) \leq \max _{\left\{\pi_{j}^{\alpha}\right\}: \sum_{j} \pi_{j}^{\alpha} \leq \bar{\pi}} \sum_{j}\left(b_{j}^{f}+\bar{c}_{j}^{\alpha}\right) \leq \sum_{j \neq i} b_{j}^{f}+\max _{\left\{\pi_{j}^{\alpha}\right\}: \sum_{j} \pi_{j}^{\alpha} \leq \bar{\pi}} \sum_{j}\left(\bar{c}_{j}^{\alpha}\right), \\
& V_{-i}(0) \leq V_{-i}\left(\pi_{i}^{f}\right)+\max _{\left\{\pi_{j}^{\alpha}\right\}: \sum_{j} \pi_{j}^{\alpha} \leq \bar{\pi}} \sum_{j}\left(\bar{c}_{j}^{\alpha}\right) .
\end{aligned}
$$

Hence, we can write:

$$
\widehat{b_{l}^{f}}=\sup _{\left\{b_{j}^{\alpha}\right\}: \pi_{j}^{\alpha} \in \Pi, j \neq i}\left(V_{-i}(0)-V_{-i}\left(\pi_{i}^{f}\right)\right) \leq \max _{\left\{\pi_{j}^{\alpha}\right\}: \sum_{j} \pi_{j}^{\alpha} \leq \bar{\pi}} \sum_{j}\left(\bar{c}_{j}^{\alpha}\right) .
$$

This implies that $\widehat{b_{l}^{f}}$ is finite, which ends the proof.

\section{Proof of Proposition 2.}

We omit the subscript $i$ in what follows. Let $\Psi \subset \Pi$ be a subset of packages on which bidder $i$ bids in the supplementary round. We represent the set of bids of bidder $i$ in the supplementary round by a disjoint union of monetary bids on all packages from the set $\Psi$, i.e., $\Phi=\left\{\left(b^{\alpha}, \pi^{\alpha}\right): \pi^{\alpha} \in \Psi\right\}$. By our convention, $\Phi$ implicitly includes all bids from the clock phase for the packages that bidder $i$ does not explicitly bid for in the supplementary phase.

Let $\Phi=\left\{\left(b^{\alpha}, \pi^{\alpha}\right)\right\}$ be such that $b^{f}<\min \left\{\widehat{b^{f}}, v^{f}\right\}$. We define marginal bids and marginal valuations as follows. Let the valuation of bidder $i$ for a package $\pi^{\alpha}$ be $v^{\alpha}$, and let his supplementary bid for this package be $b^{\alpha}$. The marginal value and marginal bid for package $\pi^{\alpha}$ are $\Delta v^{\alpha}=\left(v^{\alpha}-v^{f}\right)$ and $\Delta b^{\alpha}=\left(b^{\alpha}-b^{f}\right)$ correspondingly. The relative bid for package $\pi^{\alpha}$ is defined as $\theta^{\alpha}=\left(\Delta b^{\alpha}-\Delta v^{\alpha}\right)$. We say that a bidder bids below his (marginal) value for package $\pi^{\alpha}$ if $\theta^{\alpha}<0$.

The relative cap rule, which restricts bids in the supplementary round, and which we have written as $b^{\alpha} \leq b^{f}+\bar{c}^{\alpha}$ in the proof of Proposition 1 , can be written as $\theta^{\alpha} \leq \bar{c}^{\alpha}-$ $\Delta v^{\alpha} \equiv \gamma^{\alpha}$, where the caps $\gamma^{\alpha}$ are recursively determined by actual bids in $\Phi$, i.e., $\gamma^{\alpha}=$ 
$\gamma^{\alpha}(\Phi)$ depends on the given set of bids. By definition, $\theta^{f} \equiv 0$ and $\gamma^{f} \equiv 0$. For a set of truthful bids, denoted by $\Phi^{0}$, where $b^{\alpha}=v^{\alpha}$ for all packages, $\theta^{\alpha}=0$ for all packages as well. Since truthful bidding in the clock phase implies that truthful bidding in the supplementary round is always allowed, it follows that $\gamma^{\alpha}\left(\Phi^{0}\right) \geq 0$ for all packages.

First, we consider a set of bids $\Phi$ which only contains non-positive relative bids, i.e., $\theta^{\alpha} \leq 0$ for all $\pi^{\alpha} \in \Psi_{i}$, and for which $b^{f}<\min \left\{{\widehat{b^{f}}}, v^{f}\right\}$. Let us consider a different set of bids $\widetilde{\Phi}=\left\{\left(\widetilde{b^{\alpha}}, \pi^{\alpha}\right)\right\}$, where $\widetilde{b^{\alpha}}=b^{\alpha}+\left(\min \left\{\widehat{b^{f}}, v^{f}\right\}-b^{f}\right)>b^{\alpha}$. This set of bids is allowed in the supplementary round because it has the same relative bids: $\widetilde{\theta^{\pi}}=\theta^{\pi} \leq 0$. We will show that $\widetilde{\Phi}$ weakly dominates $\Phi$.

Similar to the analysis of VCG mechanisms, either set $\widetilde{\Phi}$ yields the same allocation as set $\Phi$ does, or set $\widetilde{\Phi}$ is winning, whereas set $\Phi$ is not: as the marginal bids are identical, it cannot be that a different package is winning under the two sets. If sets $\widetilde{\Phi}$ and $\Phi$ yield the same allocation, bidder $i$ pays the same price and gets the same surplus, whereas other bidders pay weakly higher price under $\widetilde{\Phi}$. If, on the other hand, $\widetilde{\Phi}$ is winning and $\Phi$ is not, it suffices to show that if $\widetilde{\Phi}$ is winning, the surplus is nonnegative. Let $\widetilde{\Phi}$ wins package $\pi^{\alpha}$ at the auction price $\widetilde{p^{\alpha}}$. Since $\widetilde{p^{\alpha}} \leq \widetilde{b^{\alpha}}$, the surplus $\widetilde{s^{\alpha}}$ of the bidder is

$$
\widetilde{s^{\alpha}}=v^{\alpha}-\widetilde{p^{\alpha}} \geq v^{\alpha}-b^{\alpha}-\left(\min \left\{\widehat{b^{f}}, v^{f}\right\}-b^{f}\right) \geq v^{\alpha}-b^{\alpha}-\left(v^{f}-b^{f}\right)=-\theta^{\alpha} \geq 0 .
$$

Thus, $\widetilde{\Phi}$ weakly dominates $\Phi$.

Suppose now that some bids in the package $\Phi$ are relatively higher than values. We denote the subset of packages with such bids by $\mathrm{A}, \mathrm{A} \subset \Psi_{i}$, i.e., $\theta^{\alpha}>0$ for $\pi^{\alpha} \in \mathrm{A}$ and $\theta^{\beta} \leq 0$ for $\pi^{\beta} \in \Psi_{i} \backslash$ A. Since $\Phi$ is allowed in the supplementary round, it must be that $\theta^{\alpha} \leq \gamma^{\alpha}(\Phi)$. We denote $\underline{\theta^{A}}=\min _{\alpha \in \mathrm{A}} \theta^{\alpha}>0$. Let

$$
z=\min \left\{\left(\min \left\{\widehat{b^{f}}, v^{f}\right\}-b^{f}\right), \underline{\theta^{A}}\right\}
$$

By our assumptions, $z>0$. Let us consider a set of bids, $\widetilde{\Phi}=\left\{\left(\widetilde{b^{\alpha}}, \pi^{\alpha}\right)\right\}$ where

$$
\widetilde{b^{\alpha}}=b^{\alpha} \text { for } \pi^{\alpha} \in \mathrm{A} \text {, and } \widetilde{b^{\beta}}=b^{\beta}+z \text { for } \pi^{\beta} \in \Psi_{i} \backslash \mathrm{A} \text {. }
$$


In other words, in $\widetilde{\Phi}$, the bid $b^{f}$ and all other bids $b^{\beta}$ that are not above (marginal) values, i.e., for which $\theta^{\beta} \leq 0$, are increased by $z$. All the other bids $b^{\alpha}$, that are above values, i.e., for which $\theta^{\alpha}>0$, remain intact. First, we show that $\widetilde{\Phi}$ is allowed by the relative cap rule, i.e., $\widetilde{\theta^{\alpha}} \leq \gamma^{\alpha}(\widetilde{\Phi})$ for all packages $\pi^{\alpha} \subset \Psi$. Then, we show that $\widetilde{\Phi}$ weakly dominates $\Phi$.

The bid $\widetilde{b^{f}}=b^{f}+z$ is always allowed. Let us take any other package $\pi^{\alpha} \neq \pi^{f}$ and let $r(\alpha)$ be the anchor round for package $\pi^{\alpha}$. Let $\pi^{\beta} \equiv \pi(r(\alpha))$ be the package bidder $i$ has bid for in clock round $r(\alpha), \pi^{\beta}=d_{i}^{r(\alpha)}$, and let $b^{\beta}$ be the actual bid in the supplementary round expressed for package $\pi^{\beta}$, i.e., $\left(b^{\beta}, \pi^{\beta}\right) \in \Phi$. According to the relative cap rule, $b^{\alpha} \leq B^{\alpha}=$ $b^{\beta}+p^{r} \cdot\left(\pi^{\alpha}-\pi^{\beta}\right)$. Depending on the signs of $\theta^{\alpha}$ and $\theta^{\beta}$, four cases are possible.

1. $\theta^{\alpha} \leq 0$ and $\theta^{\beta} \leq 0$. In this case, $\widetilde{b^{\alpha}}=b^{\alpha}+z$ and $\widetilde{b^{\beta}}=b^{\beta}+z$, so that

$$
\widetilde{b^{\alpha}}=b^{\alpha}+z \leq B^{\alpha}+z=b^{\beta}+\mathrm{z}+p^{r} \cdot\left(\pi^{\alpha}-\pi^{\beta}\right)=\widetilde{b^{\beta}}+p^{r} \cdot\left(\pi^{\alpha}-\pi^{\beta}\right)=\widetilde{B^{\alpha}} .
$$

Hence, bid $\widetilde{b^{\alpha}}$ satisfies the relative cap rule and is allowed.

2. $\theta^{\alpha}>0$ and $\theta^{\beta} \leq 0$. In this case, $\widetilde{b^{\alpha}}=b^{\alpha}$ and $\widetilde{b^{\beta}}=b^{\beta}+z$, so that $\widetilde{b^{\alpha}}<\widetilde{B^{\alpha}}$, and bid $\widetilde{b^{\alpha}}$ is allowed.

3. $\theta^{\alpha}>0$ and $\theta^{\beta}>0$. In this case, $\widetilde{b^{\alpha}}=b^{\alpha}$ and $\widetilde{b^{\beta}}=b^{\beta}$, so that again $\widetilde{b^{\alpha}} \leq \widetilde{B^{\alpha}}$, and bid $\widetilde{b^{\alpha}}$ is allowed.

4. $\theta^{\alpha} \leq 0$ and $\theta^{\beta}>0$. In this case, $\theta^{\beta} \geq \underline{\theta^{A}}, \widetilde{b^{\alpha}}=b^{\alpha}+z$ and $\widetilde{b^{\beta}}=b^{\beta}$. Truthful bidding, i.e., switching from $\pi^{\alpha}$ to $\pi^{\beta}$ in clock round $r$ at prices $p^{r}$ implies that $v^{\alpha}-$ $p^{r} \cdot \pi^{\alpha} \leq v^{\beta}-p^{r} \cdot \pi^{\beta}$, and, therefore,

$$
\Delta v^{\alpha}-\Delta v^{\beta}=v^{\alpha}-v^{\beta} \leq p^{r} \cdot\left(\pi^{\alpha}-\pi^{\beta}\right)
$$

Hence, the relative price cap implies

$$
B^{\alpha}=b^{\beta}+p^{r} \cdot\left(\pi^{\alpha}-\pi^{\beta}\right) \geq b^{\beta}+\Delta v^{\alpha}-\Delta v^{\beta} .
$$

Thus, $\widetilde{B^{\alpha}}-\widetilde{b^{\alpha}}=B^{\alpha}-b^{\alpha}-z$ satisfies the following inequality:

$$
\widetilde{B^{\alpha}}-\widetilde{b^{\alpha}} \geq b^{\beta}+\Delta v^{\alpha}-\Delta v^{\beta}-b^{\alpha}-z=\theta^{\beta}-z-\theta^{\alpha} \geq \theta^{\beta}-z \geq \underline{\theta^{A}}>0,
$$

which implies that $\widetilde{b^{\alpha}}$ is allowed. 
It remains to show that $\widetilde{\Phi}$ weakly dominates $\Phi$. Similar to the previous case (when $A=\emptyset$ ), if $\widetilde{\Phi}$ yields the same allocation as $\Phi$ does, it yields equal pay-off to bidder $i$ and (weakly) lower pay-offs to other bidders. If $\Phi$ is not winning but $\widetilde{\Phi}$ is winning, the very same arguments apply to show that $\widetilde{\Phi}$ yields strictly positive pay-off. The only difference here is that $\widetilde{\Phi}$ can win a different package than $\Phi$. Since only bids $b^{\beta}$ for $\pi^{\beta} \in \Psi \backslash$ A have increased, the only possibility is when $\Phi$ wins package $\pi^{\alpha} \in A$ whereas $\widetilde{\Phi}$ wins package $\pi^{\beta} \in \Psi \backslash A$.

We use the notation $V_{-i}(\pi)$ from the proof of Proposition 1, which stands for the maximum of the sum of bids of all other bidders for the remaining package $(\bar{\pi}-\pi)$. When bidder $i$ wins package $\pi^{\alpha}$, he pays auction price $p^{\alpha}=V_{-i}(\varnothing)-V_{-i}\left(\pi^{\alpha}\right)$, and gets the surplus of

$$
s^{\alpha}=v^{\alpha}-p^{\alpha}=v^{\alpha}+V_{-i}\left(\pi^{\alpha}\right)-V_{-i}(\varnothing) .
$$

Similarly, when he wins package $\pi^{\beta}$, he pays auction price $\widetilde{p^{\beta}}=V_{-i}(\varnothing)-V_{-i}\left(\pi^{\beta}\right)$, and gets the surplus of

$$
\widetilde{s^{\beta}}=v^{\beta}-\widetilde{p^{\beta}}=v^{\beta}+V_{-i}\left(\pi^{\beta}\right)-V_{-i}(\varnothing) .
$$

Moreover, it follows that $\widetilde{b^{\beta}}+V_{-i}\left(\pi^{\beta}\right) \geq \widetilde{b^{\alpha}}+V_{-i}\left(\pi^{\alpha}\right)$, since $\widetilde{b^{\alpha}}$ is not winning anymore. Using this inequality, the difference $D=\widetilde{s^{\beta}}-s^{\alpha}$ can be written as follows:

$$
D=v^{\beta}+V_{-i}\left(\pi^{\beta}\right)-v^{\alpha}-V_{-i}\left(\pi^{\alpha}\right) \geq v^{\beta}-\widetilde{b^{\beta}}+\widetilde{b^{\alpha}}-v^{\alpha} .
$$

Finally, as $\widetilde{b^{\beta}}=b^{\beta}+z$ and $\widetilde{b^{\alpha}}=b^{\alpha}$, we get:

$$
D \geq v^{\beta}-b^{\beta}-z+b^{\alpha}-v^{\alpha}=\theta^{\alpha}-z-\theta^{\beta} \geq-\theta^{\beta} \geq 0 .
$$

Thus, $\widetilde{\Phi}$ weakly dominates $\Phi$.

\section{Proof of Proposition 3.}

After all bids with $b^{f}<\widehat{b^{f}}$ are eliminated for all bidders, each bidder $i$ bids $b_{i}^{f} \geq \widehat{b_{\imath}^{f}}$ and wins a package, let it be package $\pi_{i}^{W}$. Under condition (4) of the proposition, only two cases are possible: either $e \cdot \pi_{i}^{W}>e \cdot \pi_{i}^{f}$ or $e \cdot \pi_{i}^{W}=e \cdot \pi_{i}^{f}$. Since bidder $i$ has no bids for any package $\pi_{i}^{\beta}$ for which $e \cdot \pi_{i}^{\beta}<e \cdot \pi_{i}^{f}$, he cannot win such a package. If $e \cdot \pi_{i}^{W}>e \cdot \pi_{i}^{f}$, i.e., bidder $i$ wins a package with more eligibility than the final package $\pi_{i}^{f}$, there must be another 
bidder $j$ who wins a package $\pi_{j}^{W}$ with less eligibility than $\pi_{j}^{f}$, which is impossible since $j$ has never bid for such packages. Therefore, it is necessarily that case that $e \cdot \pi_{i}^{W}=e \cdot \pi_{i}^{f}$, which implies that $\pi_{i}^{W}=\pi_{i}^{f}$, as, by condition (4), $\pi_{i}^{f}$ is the only package that bidder $i$ bids for with this number of eligibility points.

Summarizing, after all bids with $b_{i}^{f}<\widehat{b_{l}^{f}}$ are eliminated, each bidder wins a package $\pi_{i}^{f}$ irrespective of bids of the others. The prices that competitors pay will necessarily depend on the bids that a bidder puts on other packages that require more eligibility. Therefore, all bids $b_{i}^{\alpha}$ which are lower than the maximal caps $B_{i}^{\alpha}$ and which are used in computing prices for at least one other bidder are dominated by $b_{i}^{\alpha}=B_{i}^{\alpha}$.

In the second round of elimination, we eliminate low bids $b_{i}^{\alpha}$ for all such packages.

\section{Proof of Proposition 4.}

Under the conditions of the proposition, the final clock round packages $\pi^{f}$ are the same independently of whether bidder $i$ ends the clock phase in round $T$ or $(T+1)$. Because the bids of all other bidders $j \neq i$ are also assumed independent of that, the KO-bid $\widehat{b^{f}}$ of bidder $i$ is also the same. Finally, since continuing bidding for package $\pi_{i}^{\alpha}$ in round $T$ relaxes the cap restrictions for bidder $i$, Proposition 3, which uses sincere previous bidding, still holds. According to Proposition 3, bidder $i$ wins his package $\pi^{f}$ and pays $\widehat{b^{f}}$ in any case. What is different is the price the others pay.

If bidder $i$ ends the clock phase one round later, in $(T+1)$, his relative cap will allow him to submit a higher relative bid on package $\pi^{\alpha}$ and, consequently, on all other packages that require more eligibility points than this package does. According to Proposition 3, this leads to higher prices that other bidders will pay. Therefore, bidder $i$ strictly prefers not switching from $\pi^{\alpha}$ to $\pi^{f}$ in round $T$.

\section{Proof of Statement 2.}

Suppose bidders bid for $d_{i}^{T}=\pi_{i}^{F}=(1,1)$ in round $T \leq 13$. We will show that at least one bidder has a profitable deviation. Let bidder $i=1$ switch from $d_{1}^{T-1}=(2,1)$ to $d_{1}^{T}=(1,1)$ 
in round $T$. We consider a deviation, denoted by a 'tilde' on all its variables, in which bidder 1 bids for $\tilde{d}_{1}^{T}=(2,1)$ in round $T$ and then switches to $\tilde{d}_{1}^{T+1}=(1,1)$.

In general, the clock may either stop in round $T+1$, or it may continue. Nevertheless, if bidder 1 wins package $(1,1)$ in this deviation (and other bidders win $(1,1)$ as well), he pays the same auction price. The reason is that with lexicographic preferences for raising rivals' cost, this price is determined by the caps of other bidders for packages $(1,1),(3,0)$, and $(0,3)$ : any other combination of bids by other bidders, such as $(2,1)$ together with $(0,2)$ or $(1,2)$ together with $(2,0)$ will necessarily lead to a lower total bid than the combination $(3,0)$ and $(0,3)$. Under our assumptions, the caps for the packages $(3,0)$ and $(0,3)$ do not change in relation to the bid on $(1,1)$ in the supplementary round. Thus, the KO-bid $\widehat{b_{1}}$ does not change, and bidders 2 and 3 make sure that bidder 1 does pay this price.

Whether bidder 1 wins $(1,1)$ or another package only depends on his relative bids for the other packages. If the development of the clock phase after the deviation allows bidder 1 to get different package with higher surplus, he will do this, and the deviation is strictly profitable. Otherwise, bidder 1 gets the same package $(1,1)$ at the same auction price as before the deviation. In the latter case, it is the surplus that other bidders get which will be different. It remains to be shown that in the deviation, if bidder 1 wins $(1,1)$, other bidders get strictly lower surplus.

Suppose, first, that other bidders win $(1,1)$. In such a case, bids of bidder 1 for packages $(3,0)$ and $(0,3)$ in the supplementary round determine the prices other bidders pay. Since bidder 1 switches from $(2,1)$ to $(1,1)$ one round later in the deviation, his caps for $(3,0)$ and $(0,3)$ become 2 and, respectively 1 higher than before the deviation. Thus, the new KO-bids of bidders 2 and 3 get increased by 2 (this is true if bidders 2 and 3 bid for $(1,1)$ in the last clock round, otherwise it is the highest price that bidders 2 and 3 may pay had they won $(1,1)$ package). Whether it results in an increase in prices they pay depends on whether the new KO-bids are below the values. It is easy to compute that for $T=13$, the highest possible KObids for bidders 2 and 3 are $\widehat{b_{2}}=\widehat{b_{3}}=38$, when all three bidders switch from $(2,1)$ to $(1,1)$ in round 13. Thus, for $T \leq 13$, the deviation results in higher prices that others pay for $(1,1)$. 
Suppose now that at least one other bidder, let it be bidder 2 , does not win $(1,1)$ after the deviation. The following cases are possible.

- If bidder 2 wins $(2,1)$ (similar for $(1,2)$ package), then the remaining bidder 3 wins nothing (no bidder ever wins $(0,1)$ or $(1,0)$ in equilibrium), and, therefore, bidder 1 wins $(1,2)$. Therefore, the supplementary bids satisfy $b_{1}^{(1,1)}+b_{2}^{(1,1)}+b_{3}^{(1,1)} \leq$ $b_{1}^{(1,2)}+b_{2}^{(2,1)}$. By bidding $b_{1}^{(1,2)}=b_{1}^{(1,1)}$, bidder 1 can ensure that (i) either he wins $(1,2)$ at the price of $(1,1)$ before deviation, or (ii) all bidders win $(1,1)$. In the former case, bidder 1 gets a higher surplus. In the latter case, by the above argument, bidders 2 and 3 will pay more.

- If bidder 2 wins nothing, then either bidder 3 wins $(2,1)$ or $(1,2)$, and the same argument applies, or bidder 1 is the only winning bidder, which cannot happen in equilibrium.

Thus, the deviation is always profitable for $T \leq 13$, and such equilibria may only exists for $T \geq 14$.

Part (b) is proven by direct verification. Let bidders keep bidding on $(2,1)$ up to (and including) round 13 and drop to $(1,1)$ in round 14 . The KO-bid is then 41 , which is above the value. Thus, bidding $b=40$ is dominant. In the second elimination round, all bids are eliminated which are both (1) below caps, and (2) are never winning when $b=40$. Since the caps allow bids with $b_{(3,0)}=b+21$, and $b_{(0,3)}=b+20$, all bids with $b_{(3,0)} \in[60,61]$ and $b_{(0,3)} \in[59,60]$ remain undominated.

\section{Proof of Proposition 5.}

Following the arguments from the proof of Proposition 2 we argue that all bids with $b_{i}^{f}<D_{i}$ are dominated by $b_{i}^{f}=D_{i}$. Thus, in any undominated equilibrium, each bidder bids at least $b_{i}^{f}=D_{i}$. Suppose that $b_{i}^{\alpha} \leq D_{i}$ for all other packages $\pi_{i}^{\alpha} \in \Psi_{i}$, and consider a bidder $j$.

If bidder $j$ bids $b_{j}^{f}=D_{j}$, he wins $\pi_{j}^{f}$ at zero price. Indeed, all other bidders together do not bid more than $D_{i}$ on larger packages. This implies that if bidder $j$ bids $b_{j}^{\alpha} \leq D_{j}$, all other 
bidders win their final clock round packages at zero price. By bidding just above the budget $b_{j}^{\alpha}=D_{j}+\varepsilon_{j}$, where $\varepsilon_{j}>0$ and $\varepsilon_{j}<\min _{i \neq j} D_{i}$, on packages $\pi_{j}^{\alpha} \neq \pi_{j}^{f}$ bidder $j$ still wins $\pi_{j}^{f}$ at zero price. However, the prices that all other bidders pay for their final clock round packages $\pi_{i}^{f}$ equal to $\varepsilon_{j}$, provided the set of packages $\Psi_{j}$ is exhaustive, $\Psi_{j}=\Pi$. Thus, there is no equilibrium where $b_{i}^{\alpha} \leq D_{i}$ for all bidders $i$.

\section{Proof of Statement 3.}

If the three bidders choose these strategies, $B^{H}, B^{D}$, and $B^{D}$, the total sum of winning bids is $35+35+35=105$. The two "Dove-like" bidders are willing to pay 100 together for the full spectrum, while one "dove-like” bidder and one "hawk-like” bidder have together also expressed a willingness to pay of 105. Each of the "dove-like” bidders pay 35 (as for each of them, the other two bidders are together willing to pay 105 for the total spectrum). The "hawk-like” bidder pays 5 less (as others are together maximally willing to pay 100 for the full spectrum - the combination $(2,1)$ and $(1,2))$.

It is not difficult to see that no bidder individually has an incentive to deviate. If by deviating, they change the final allocation, at least one bidder does not get any spectrum. If this is the deviating bidder, he strictly gets a smaller pay-off than in the proposed equilibrium. If it is another bidder, who does not win spectrum after the deviation, then the deviating player has to pay the opportunity cost - which is what the losing bidder expressed to be willing to pay for a larger package - which is more than the budget constraint (and valuation). Finally, as bidders do not affect their own payment, if the final allocation is not affected and in the equilibrium they already bid so as to maximally increase others' payments without affecting the allocation, deviating can only result in payments of other bidders that are not larger. Thus, no deviation is profitable.

\section{Appendix II: Final Cap Rule}

Ausubel and Cramton (2011) have proposed a new rule that imposes more constraints on bids in the supplementary rounds. They require that all bids on packages that require more 
eligibility points than the bid in the final clock round package, satisfy a revealed preference constraints with respect to all rounds after the relevant anchor round where the bidders reduced eligibility points, including the final round as well. They term this rule the Simplified Revealed Preference Cap. In several recent CCA designs, for example in Australia and Canada, this Simplified Revealed Preference Cap, or final cap rule, has been adopted in practice. Under this rule, all bids $b_{\alpha}$ for packages $\alpha$ in the supplementary round should satisfy the following condition:

$$
b^{\alpha} \leq B^{\alpha}=b^{f}+p^{T} \cdot\left(\pi^{\alpha}-\pi^{f}\right) .
$$

Ausubel and Cramton (2011) show that this rule implies that bidders win the package they were bidding for in the final clock round if the clock phase ends without excess supply (like in our example). The rule implies that if bidders bid truthfully in the clock phase, bidders cannot bid more than respectively $b+7, b+5, b+9$, and $b+3$ on the packages $(2,1)$, $(1,2),(3,0)$, and $(0,3)$. As a result, no two bidders can outbid the other remaining bidder. For the total supply of $(3,3)$, they together cannot bid more than 12 more than they jointly bid on the supply of $(2,2)$. Thus, the final clock bid of $b=12$ is sufficient to win $(1,1)$, and the total payment is not more than 12, substantially reducing bidders’ possible payments.

Knowing that bids $b+7, b+5, b+9$, and $b+3$ on the packages $(2,1),(1,2),(3,0)$, and $(0,3)$ cannot be winning, players can submit these higher bids without any risk and make sure that rivals pay 12 for their winning package $(1,1)$. Thus, under the final cap rule as well, bidders can game and bid without any risk on packages they do not value intrinsically. This consideration has led the UK regulator OFCOM to withdraw the final cap rule from its auction design and to revert back to the relative cap rule. ${ }^{30}$

Our analysis shows few important differences between the relative and final cap rules. First, the equilibrium payments under the relative cap rule are, in our example, almost twice as high as under the final cap rule. Second, it is true that by bidding on $(3,0)$ and $(0,3)$, the bidder runs a risk, under the relative cap rule, to obtain these packages that do not have an

30 See, OFCOM (2012), Assessment of future mobile competition and award of $800 \mathrm{MHz}$ and 2.6 GHz, 24 July 2012. 
intrinsic value. This risk is absent under the final cap rule. However, the above iterative dominance argument shows that also under the relative cap rule, this risk is minimal knowing that rational rivals will try to avoid any risk of not winning any package and, therefore, will bid higher on $(1,1)$ than what others together can add to their total combined bid for $(2,2)$.

If bidders bid truthfully in the clock phase, the trade-off is then clear: under the final cap rule, the potential for gaming in the supplementary round is severely restricted, but gaming is without any risk. Under the relative cap rule, the potential for gaming is very large, but carries some risk (that depends on whether other players protect themselves against this gaming by rivals). There is, thus, no clear-cut case to be made for either one of these rules. In particular, it is not the case that under the relative cap rule, gaming will not arise, and if it arises, it can have a severe impact on auction prices.

Finally, and related, the relative cap rule has the disadvantage that the outcome of the auction may be inefficient. If two out of the three players follow the logic of iterative dominance and bid $B=(23,30,34,32,37)$ on the five possible packages, whereas the other bidder bids strictly less than 23 on $(1,1)$, the outcome is that there are two winners who win packages $(3,0)$ and $(0,3)$ which they do not have any intrinsic value for.

If bidders want to raise rivals' cost in a CCA under the final cap rule further, they can behave strategically in the clock phase. The reasons are the same as before (and potentially stronger) as to get around the severe restrictions of the supplementary round bids, bidders demand larger packages in the clock phase than they would normally do in truthful bidding.

To see this, suppose that all bidders start the clock phase in the same way as before, but continue bidding on $(2,1)$ until the prices reach the amount $(x, 5)$, for some $x$ and then drop demand to $(1,1)$ finishing off the clock phase. Knowing that the maximum bids on the packages $(2,1),(1,2),(3,0)$, and $(0,3)$ are then given by $b+x, b+5, b+2 x-5$, and $b+10-x$, competitors can together bid maximally $2 b+x+5$ on the total supply, if they together bid $2 b$ on two blocks in each band. Thus, by choosing a large $x$, bidders can also raise rivals' cost under the final cap rule. 


\begin{tabular}{|c|c|c|c|c|c|c|c|}
\hline \multirow{2}{*}{$\begin{array}{c}\text { Scenario } \\
\text { no. }\end{array}$} & \multicolumn{2}{|c|}{ Round 1 } & \multicolumn{2}{c|}{ Rounds 2-7 } & \multicolumn{2}{c|}{ Round 8 } & KO- \\
\cline { 2 - 7 } bid \\
\hline 1 & Bidder 2 & Bidder 3 & Bidder 2 & Bidder 3 & Bidder 2 & Bidder 3 & 28 \\
\hline 2 & $(2,1)$ & $(0,3)$ & $(3,0)$ & $(1,2)$ & $(1,0)$ & $(1,2)$ & 28 \\
\hline 3 & $(2,1)$ & $(0,3)$ & $(3,0)$ & $(1,2)$ & $(0,1)$ & $(2,1)$ & 28 \\
\hline 4 & $(1,2)$ & $(1,2)$ & $(3,0)$ & $(1,2)$ & $(0,1)$ & $(2,1)$ & 28 \\
\hline 5 & $(2,1)$ & $(0,3)$ & $(2,1)$ & $(2,1)$ & $(0,1)$ & $(2,1)$ & 24 \\
\hline 6 & $(1,2)$ & $(1,2)$ & $(2,1)$ & $(2,1)$ & $(0,1)$ & $(2,1)$ & 23 \\
\hline 7 & $(2,1)$ & $(0,3)$ & $(3,0)$ & $(1,2)$ & $(2,0)$ & $(0,2)$ & 28 \\
\hline 8 & $(1,2)$ & $(1,2)$ & $(3,0)$ & $(1,2)$ & $(2,0)$ & $(0,2)$ & 29 \\
\hline 9 & $(2,1)$ & $(0,3)$ & $(2,1)$ & $(2,1)$ & $(2,0)$ & $(0,2)$ & 24 \\
\hline 10 & $(1,2)$ & $(1,2)$ & $(2,1)$ & $(2,1)$ & $(2,0)$ & $(0,2)$ & 23 \\
\hline 11 & $(2,1)$ & $(0,3)$ & $(3,0)$ & $(1,2)$ & $(1,1)$ & $(1,1)$ & 28 \\
\hline 12 & $(1,2)$ & $(1,2)$ & $(3,0)$ & $(1,2)$ & $(1,1)$ & $(1,1)$ & 29 \\
\hline 13 & $(2,1)$ & $(0,3)$ & $(2,1)$ & $(2,1)$ & $(1,1)$ & $(1,1)$ & 24 \\
\hline 14 & $(1,2)$ & $(1,2)$ & $(2,1)$ & $(2,1)$ & $(1,1)$ & $(1,1)$ & 23 \\
\hline
\end{tabular}

Table 3. Clock phase scenarios.

\section{Appendix III: Computing KO-bid}

There are 14 different scenarios (up to the symmetry of bidders 2 and 3) of the clock phase development that generate the same aggregate demand as Table 2 presents, conditional on bidding behavior of bidder 1 . Table 3 presents all 14 different ways of individual bidding of bidders 2 and 3. Scenario 14 is the actual clock phase development.

For each scenario, we can compute KO-bid according to Proposition 1, these bids are shown in the last column. As one can see, the maximal KO-bid is 29, and it occurs in scenarios 2, 8, and 12. Thus, when the clock phase development is not public information, Proposition 1 remains valid, and the KO-bid must be calculated as a maximum KO-bid across all possible scenarios. The same technique, although with a much larger set of scenarios, can be used to compute KO-bids if bidders only get information about clock prices, i.e., in which bands there is an excess demand. 